\title{
A Hybrid Systematic Review Approach on Complexity Issues in Data-Driven Fuzzy Inference Systems Development
}

\author{
Diana KALIBATIENE $\dot{1}^{1}$, Jolanta MILIAUSKAITÉ ${ }^{2, *}$ \\ ${ }^{1}$ Department of Information Systems, Faculty of Fundamental Sciences, \\ Vilnius Gediminas Technical University, Vilnius, Lithuania \\ ${ }^{2}$ Institute of Data Science and Digital Technologies, Cyber-Social Systems Engineering Group, \\ Vilnius University, Vilnius, Lithuania \\ e-mail:diana.kalibatiene@vilniustech.lt,jolanta.miliauskaite@mif.vu.lt
}

Received: September 2020; accepted: January 2021

\begin{abstract}
The data-driven approach is popular to automate learning of fuzzy rules and tuning membership function parameters in fuzzy inference systems (FIS) development. However, researchers highlight different challenges and issues of this FIS development because of its complexity. This paper evaluates the current state of the art of FIS development complexity issues in Computer Science, Software Engineering and Information Systems, specifically: 1) What complexity issues exist in the context of developing FIS? 2) Is it possible to systematize existing solutions of identified complexity issues? We have conducted a hybrid systematic literature review combined with a systematic mapping study that includes keyword map to address these questions. This review has identified the main FIS development complexity issues that practitioners should consider when developing FIS. The paper also proposes a framework of complexity issues and their possible solutions in FIS development.
\end{abstract}

Key words: membership function, fuzzy rule, fuzzy inference system, issue, limitation, complexity, systematic literature review, systematic mapping.

\section{Introduction}

Fuzzy sets have been used greatly in different scientific areas. In their study, authors of Kahraman et al. (2016) found many application areas in theoretical and practical studies, like engineering, arts, humanities, computer sciences, health science, life sciences, physical sciences, etc. They present a comprehensive literature review on the fuzzy set theory realization covering 50 years since Zadeh had proposed it in 1965. The authors have not analysed any complexity issues of the fuzzy set theory application and realization. However, the authors have highlighted the need for a standard notation of the fuzzy set theory. Various authors frequently encounter different notations for the same concepts

\footnotetext{
*Corresponding author.
} 
of the theory in their publications. A standard notation will improve the theory's value and will be a significant step to provide the condition that has already been implemented in the classical logic. Another problem defined by the authors of Kahraman et al. (2016) is the segregation between classical logic publications and fuzzy logic publications. Moreover, there can be found too many different fuzzy models and approaches in the literature without sufficient discussions about their correctness or deficiencies that may create a doubtful point of view to the theory (Kahraman et al., 2016). One of the main reasons for these problems is the complexity of the fuzzy set theory and its application in different areas. Therefore, there is a need for a more comprehensive study of the fuzzy inference systems development complexity issues and their systematization in this context.

We can find plenty of approaches to develop FIS in the literature automatically (RuizGarcia et al., 2019; Lee, 2019; Mirko et al., 2019; Askari, 2017). Authors of these approaches point out various limitations, issues, or drawbacks of developing FIS because of its complexity, like the sparse rule base (RB) (Antonelli et al., 2010), high dimensional data (Alcalá et al., 2009b), a vast number of linguistic terms (Askari, 2017; Ephzibah, 2011), etc. All those complexity issues complicate the automatic development of FIS and need to be solved. However, in the fuzzy set theory application field, they are not investigated sufficiently in a systematic and comprehensive way. Authors of the analysed papers have focused on solving a particular task, like reducing computational complexity through decreasing the number of fuzzy rules (Ruiz-Garcia et al., 2019; Zhu et al., 2017; Harandi and Derhami, 2016; Bouchachia and Vanaret, 2014) or reducing MFs (Fan et al., 2019; Ibarra et al., 2015), etc. This lack of understanding of a general situation hampers progress in the analysed field since academics offer limited approaches (Ivarsson and Gorschek, 2011).

Consequently, the following research questions arise: 1) "What complexity issues exist in the context of developing fuzzy inference systems (FIS)?" (RQ1) and 2) "Is it possible to systematize existing solutions of identified complexity issues?" (RQ2). In order to answer the defined research questions, this paper presents a synergy of two well-known research methods - a systematic literature review (SLR) and a systematic mapping survey (SMS). SLR is used to perform in-depth analysis and obtain answers for the defined research questions (Kitchenham et al., 2009; Mallett et al., 2012), and SMS with a keyword map - for providing more general research trends and detecting topics that exist within the analysed field (Petersen et al., 2015; Kitchenham et al., 2011; Ramaki et al., 2018). Moreover, a keyword map allows us to visualize (Linnenluecke et al., 2019) and better understand each concept's real meaning in FIS development, systematize existing solutions of identified complexity issues, and develop the framework of complexity issues and their possible solutions in FIS development.

This research increases the body of knowledge on FIS development theory by providing a systematic view of complexity issues and their existing solutions. New trends in developing FIS are uncovered as well. Additionally, the results of this research can help researchers and practitioners become familiar with found FIS complexity issues and their possible solutions. Our main scientific contribution and advantages of this paper are as follows: 
1. The complexity issues in FIS development are found and systematized.

2. The solutions for the found complexity issues in FIS development are discovered.

3. The framework of FIS development complexity issues and their solutions is proposed.

4. The hybrid SLR and SMS approach with a keyword map is employed to answer the research questions at various depths.

The novelty of this research is the systematic view of the found complexity issues in FIS development, the proposed framework of FIS development complexity issues and their solutions. The rest of this paper is structured as follows. Section 2 introduces the main concepts and explains their use in the paper. Section 3 presents related works. Section 4 presents the review method. Section 5 shows the obtained results of hybrid systematic review approach on complexity issues. Section 6 provides the developed framework of FIS development complexity issues and their possible solutions. At last, the discussion is drawn in Section 7, and conclusions in Section 8.

\section{Background}

Here, the concept of complex is relevant to the context of software systems. Odell (2002) defines a system as complex, if it cannot be fully understood by analysing its components, i.e. the interaction among the components must also be considered. As Battram (1998) defines: "Complexity refers to the condition of the universe which is integrated and yet too rich and varied for us to understand in simple common ... ways. We can understand many parts of the universe in these ways, but the larger more intricately related phenomena can only be understood by principles and patterns — not in detail." According to Corning (1998), Benigni et al. (2012), a system is complex, if it exhibits the following properties: 1) it is composed of many interconnected parts; 2) the parts are highly interdependent; and 3) their behaviour produces synergies (combined effects) that are not easy to predict from the behaviour of the individual parts by analysing them in isolation. Here, we understand a system to be complex if it consists of a number of components interacting with each other in different ways that allow new properties to emerge (Waldrop, 1993).

Further, we analyse the complexity of a fuzzy inference system (FIS) that uses fuzzy set theory to map inputs to outputs and consists of the following main components (Askari, 2017; Mamdani, 1974; Takagi and Sugeno, 1985; Miliauskaitè and Kalibatiene, 2020a) (see Fig. 1). Fuzzification is converting the data input into fuzzy linguistic variables by applying a particular fuzzification method. This method presents a way of determining the degree to which input variables belong to each of the appropriate fuzzy sets via the membership functions (MFs). Fuzzy Inference involves applying a set of (fuzzy) rules from a Knowledge base to fuzzy inputs in order to generate a set of fuzzy outputs. Defuzzification translates the obtained fuzzy output for the final user into an understandable crisp output, using a particular defuzzification method.

In a data-driven FIS, the underlying MFs and fuzzy rules are generated automatically from synthetic or real data streams but not defined by an expert manually (Miliauskaite and Kalibatienè, 2020b) (Fig. 1). 


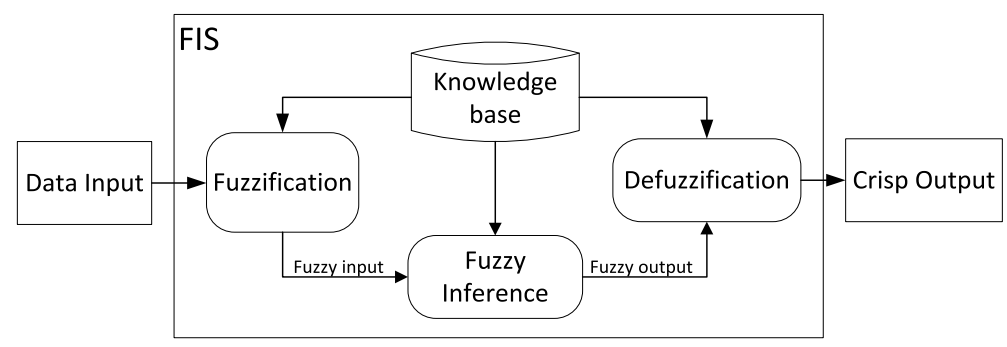

Fig. 1. The reference schema of FIS.

\section{Related Works}

Authors of Antonelli et al. (2011) understand complexity as interpretability of rule base (RB), and interpretability of fuzzy partitions as integrity of the database. Data complexity is measured in terms of the average number of patterns per variable (i.e. data density) for pattern recognition (Ephzibah, 2011). FIS suffers from exponential complexity, manifested through some linguistic terms (number of subspaces on the universe of discourse of input variables) and some input variables (Askari, 2017). Complexity is also measured by counting the number of operations (Ephzibah, 2011) or the number of RB elements, including the number of MFs, rules, premises, linguistic terms, etc. (Askari, 2017). Selecting a small number of right linguistic terms is essential for better interpretability. The total number of parameters of the fuzzy RB is also a measure of interpretability. A system with a smaller number of parameters is more interpretable and less complex (Ishibuchi and Nojima, 2009). In Askari (2017), Kaynak et al. (2002), authors suggest reducing the exponential complexity of FIS by reducing the number of fuzzy (linguistic) terms or the number of fuzzy (linguistic) variables or both. The model interpretability is measured in terms of complexity (Antonelli et al., 2016): "Complexity is affected by the number of features used for generating the model: the lower the number of features, the lower the complexity". RB complexity is measured as the total number of conditions in the rules' antecedents (Alcalá et al., 2009b).

The most related reviews to the present review are summarized in Table 1 . The authors of Liu et al. (2017) have suggested developing hierarchical structure-based vague reasoning algorithms to handle complex systems and to reduce its complexity through decomposition and reuse of the whole system. Other findings in Liu et al. (2017) are related to the need of the adoption of existing knowledge inference systems for big data and real-time applications, increasing the accuracy of models while reducing reasoning efficiency and computational cost. Authors of D'Urso (2017) have stated that the higher type fuzzy systems are increasingly complex; therefore, they only focus on type-2 fuzzy sets to reduce the computational complexity. The authors of Shahidah et al. (2017) note the importance of a fuzzy system in correlated node behaviour detection with emphasis on the enhancement of the computational complexity and accuracy detection. In SanchezRoger et al. (2017), authors have mentioned uncertainty of information in the complex environment of the financial field, where fuzzy logic helps to manage those complexities. 
Table 1

Summary of the analysed literature reviews on complexity issues in FIS.

\begin{tabular}{|c|c|c|c|c|}
\hline Reference & Research method & Research domain & Complexity issues & Solution/Conclusion \\
\hline Liu et al. (2017) & $\begin{array}{l}\text { Literature review, } \\
\text { RM not presented }\end{array}$ & $\begin{array}{l}\text { Fuzzy Petri nets } \\
\text { (FPN) for knowledge } \\
\text { representation }\end{array}$ & $\begin{array}{l}\text { FPN algorithm } \\
\text { complexity increases } \\
\text { and depends on the } \\
\text { scale of the created } \\
\text { FPN model }\end{array}$ & $\begin{array}{l}\text { Hierarchical } \\
\text { structure based } \\
\text { reasoning } \\
\text { algorithms, } \\
\text { combination of FPN } \\
\text { with other } \\
\text { uncertainty theories }\end{array}$ \\
\hline D’Urso (2017) & $\begin{array}{l}\text { SLR, RM not } \\
\text { presented }\end{array}$ & Clustering approaches & $\begin{array}{l}\text { Type complexity, } \\
\text { computation } \\
\text { complexity }\end{array}$ & $\begin{array}{l}\text { Type reduction, } \\
\text { interval type- } 2 \text { fuzzy } \\
\text { sets }\end{array}$ \\
\hline $\begin{array}{l}\text { Shahidah et al. } \\
\text { (2017) }\end{array}$ & $\begin{array}{l}\text { SLR, RM } \\
\text { (Kitchenham et } \\
\text { al., 2009) }\end{array}$ & $\begin{array}{l}\text { Node behaviour } \\
\text { detection in wireless } \\
\text { sensor network }\end{array}$ & $\begin{array}{l}\text { Computational } \\
\text { complexity }\end{array}$ & $\begin{array}{l}\text { General conclusions, } \\
\text { automated } \\
\text { uncertainty based } \\
\text { fault detection and } \\
\text { diagnosis approach }\end{array}$ \\
\hline $\begin{array}{l}\text { Sanchez-Roger } \text { et } \\
\text { al. (2017) }\end{array}$ & $\begin{array}{l}\text { SLR, bibliometric } \\
\text { analysis }\end{array}$ & Financial field & $\begin{array}{l}\text { Uncertainty of } \\
\text { information, complex } \\
\text { environment }\end{array}$ & General conclusions \\
\hline $\begin{array}{l}\text { Rajab and } \\
\text { Sharma (2018) }\end{array}$ & Review & $\begin{array}{l}\text { Neuro-fuzzy systems } \\
\text { in business }\end{array}$ & $\begin{array}{l}\text { The mass and } \\
\text { vagueness of datasets, } \\
\text { complex/uncertain/un- } \\
\text { clear/lack real world } \\
\text { information }\end{array}$ & General conclusions \\
\hline
\end{tabular}

The authors Rajab and Sharma (2018) have found in their review that for improving the performance of neuro-fuzzy systems (NFS), various data pre-processing methods, higher order neuro-fuzzy methodologies and various optimization mechanisms were combined with these systems to improve the overall performance. In future, newer efficient input and output processing techniques and different optimization techniques can be applied with various NFS approaches (Rajab and Sharma, 2018).

However, the complexity issues of FIS development are not analysed in detail in the presented papers. Consequently, there is no systematic and comprehensive review of FIS complexity issues and their solutions, i.e. the analysed papers differ from this study by research questions and their aim.

\section{Review Method}

SLR was adopted as proposed by Kitchenham et al. (2009), and SMS from Petersen et al. (2015), Kitchenham and Charters (2007), Linnenluecke et al. (2019), Ramaki et al. (2018). The hybrid SLR and SMS approach used in this review is presented in Fig. 2. It consists of four main stages: review design, review conduct, review analysis, and quality assurance (i.e. reducing threats to validity). The first, second, and third stages are done sequentially one after the other with some iterations, if necessary, and the fourth stage is 


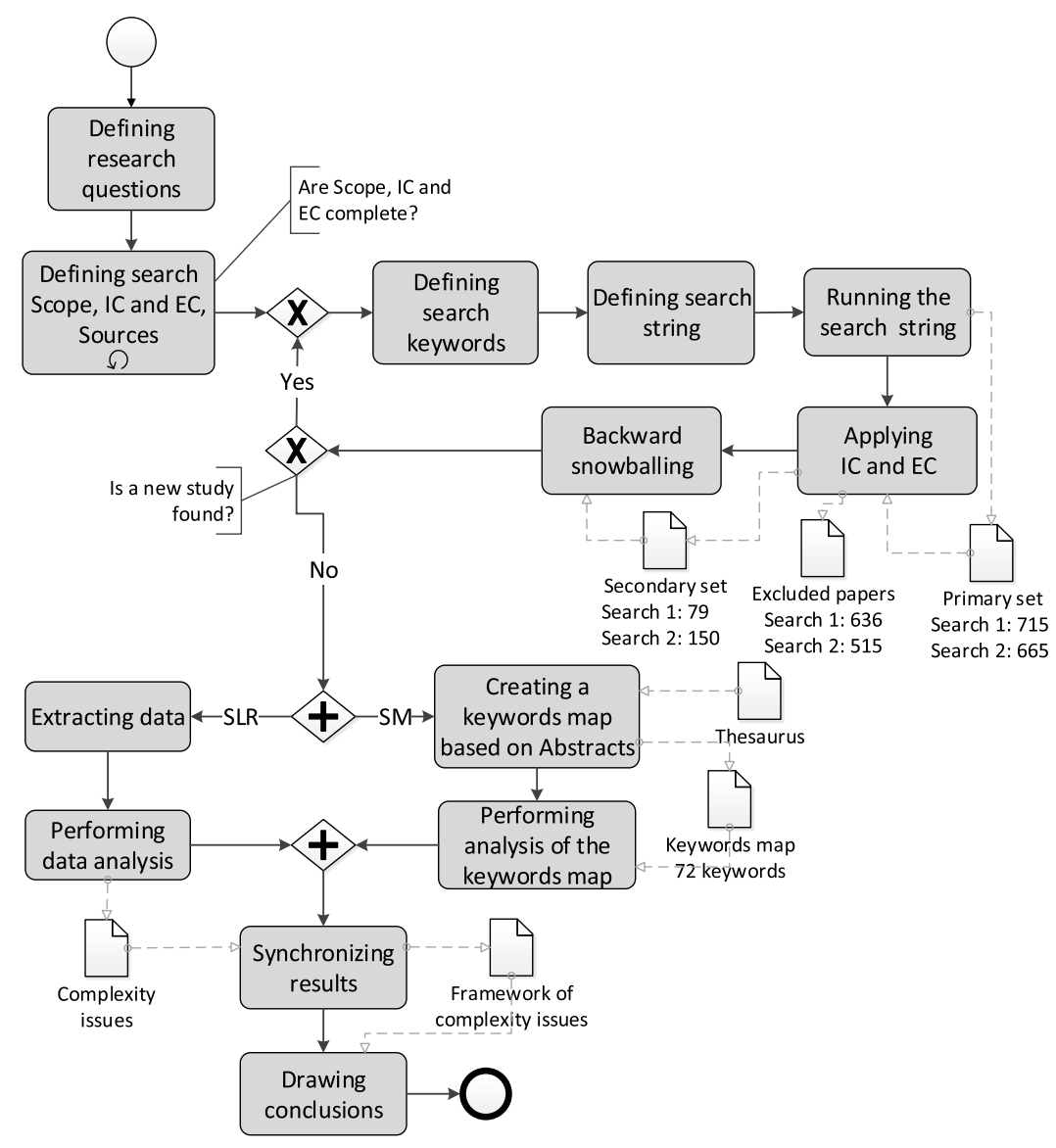

Fig. 2. The hybrid SLR and SMS approach schema.

interleaved with the first, second, and third stages. In the rest of this section, the details of those stages are described.

Review design consists of the following activities:

1. Defining research question. The review begins from the definition of the research questions, which were described in Introduction. This review was conducted from December 2019 until April 2020, so we considered the papers that have been published until January 2020.

2. Defining research scope, inclusion $(I C)$ and criteria $(E C)$, search sources. The scope of our review is all papers that have been prepared in Computer Science (CS), Information Systems (IS), and Software Engineering (SE). Web of Science (WoS) database was chosen as the search source. For more motivation, see Section "Source evaluation".

Based on the defined scope of the research, papers' IC and EC are defined as the following:

IC1: Universally accepted relevant works on FIS development, including MFs and fuzzy rules development, construction or generation, issues, limitations or complexity. 
Table 2

Keyword hierarchy.

\begin{tabular}{lll}
\hline Main concepts & Reduced concepts & Rationale \\
\hline $\begin{array}{l}\text { Fuzzy Inference System } \\
\text { Membership function }\end{array}$ & $\begin{array}{l}\text { fuzzy* } \\
\text { "membership function*" } \\
\text { "fuzzy rule*" }\end{array}$ & $\begin{array}{l}\text { Specifies overall area of developing and using FIS. } \\
\text { FIS involves generation, construction and } \\
\text { development of MFs and fuzzy rules. }\end{array}$ \\
$\begin{array}{l}\text { Development } \\
\text { Generation }\end{array}$ & $\begin{array}{l}\text { develop* } \\
\text { generat* } \\
\text { Construction }\end{array}$ & $\begin{array}{l}\text { FIS involves generation, construction and } \\
\text { development of MFs and fuzzy rules. }\end{array}$ \\
$\begin{array}{l}\text { Complexity issue } \\
\text { Issue } \\
\text { Complex }\end{array}$ & $\begin{array}{l}\text { issue* } \\
\text { complex* }\end{array}$ & Those concepts are primary from the RQs. \\
\hline
\end{tabular}

Note: some general terms, which refer to FIS development and complexity issues, like data, input, output, etc., were excluded, since their inclusion into the search string increased the number of papers found, but additional papers did not expand the knowledge provided on the research topic. Therefore, they were excluded from the search but are still found together with the keywords used.

IC2: Papers must be open access.

EC1: Exclude papers that do not relate to the FIS development complexity issues, i.e. papers that contain relevant keywords, but FIS issues, limitations or complexity are not discussed in the abstract.

EC2: Exclude duplicate papers that repeat ideas described in earlier works and their abstracts are similar, i.e. if one paper is an extension of another, the less extended (i.e. containing less pages) paper is excluded (Kitchenham, 2004).

EC3: Exclude papers, whose length is less than 10 pages, since such short papers can present only a general idea, but cannot describe the overall approach (Dybå and Dingsøyr, 2008).

EC4: Exclude grey literature, conference cover, posters, and so on (Dybå and Dingsøyr, 2008).

EC5: Exclude papers not in English.

3. Defining search keywords. In this step, we define relevant keywords, which together with selected sources are going to be used to formulate a search string. The following keyword hierarchy was established in Table 2.

4. Defining search string. Here, we have specified the following two search strings:

Search string 1: ((fuzzy*) AND ("membership function*”) AND ("develop*" OR "generat*" OR “construct*”) AND ("issue*" OR "limit*" OR "complex*")).

Search string 2: (("fuzzy rule*”) AND ("issue*" OR "limit*" OR “complex*”) AND ("reduc*" OR "optimiz*")).

Search string 1 is the primary search string used for the initial search. Search string 2 is the secondary search string developed using keywords from Table 2 and refined, by adding reduc* and optimiz*, after backward snowballing.

Review conduct. As shown in Fig. 2, it consists of the following activities:

5. Running the search string. In this step, the defined search strings are running in WoS engine. For the first iteration, we have used the search string 1 . The output of this search is a primary set of the papers. For the second iteration, we have used the search 
Table 3

Number of obtained papers (Articles (A) or Proceedings Papers (PP)).

\begin{tabular}{|c|c|c|c|c|c|c|c|c|}
\hline \multirow[t]{2}{*}{ Search } & \multicolumn{4}{|c|}{ The primary set of papers } & \multicolumn{4}{|c|}{ The secondary set of papers } \\
\hline & Years & A & $\mathrm{PP}$ & All & Years & A & $\mathrm{PP}$ & All \\
\hline Search1 & 1991-2019 & 437 & 278 & 715 & 1993-2019 & 74 & 5 & 79 \\
\hline Search2 & 1991-2019 & 366 & 332 & 665 & 1993-2019 & 147 & 3 & 150 \\
\hline All & 1991-2019 & 803 & 610 & 1380 & 1993-2019* & $209 *$ & $8^{*}$ & $217 *$ \\
\hline
\end{tabular}

*Duplicate papers are excluded.

string 2, which was developed after applying backward snowballing strategy. The results of the search are presented in Table 3.

6. Applying IC and EC. Here, the predefined IC and EC were applied to the primary set to obtain only relevant papers on the analysed topic. The input of this step is the primary set of papers. The output is the secondary set of papers. The results of the application of IC and EC are presented in Table 3.

7. Backward snowballing. In this review, we have applied backward snowballing technique (Jalali and Wohlin, 2012) for searching relevant keywords in titles, abstracts and keywords of the secondary set of papers to improve and refine search strings. As the result of the first iteration, the secondary search string (Search string 2) was developed.

The direct inclusion of the backward snowballing technique in the review method allows us to iteratively evaluate the secondary set of papers. It supplements the search process with additional keywords, including new relevant papers that may be omitted due to too many papers. The backward snowballing technique should be applied until new papers are found or time runs out. In this review, two iterations were performed, since new iterations do not increase the number of relevant papers.

Review analysis. As shown in Fig. 2, the data (i.e. the primary set of complexity issues) analysis consists of two parallel branches, i.e. SLR and SMS. It consists of the following activities:

8. Extracting data. This step is conventional to the data extraction in SLR (Petersen et al., 2015). It covers extracting initial data from Abstracts and tabulating it. The input of this step is Abstracts of the secondary set of papers. The output is the primary set of complexity issues (see Fig. 4).

9. Performing data analysis. This step covers the analysis of the extracted data. The data extracted from the papers was tabulated and plotted to present basic information on RQs. The obtained initial set of complexity issues was grouped into categories, based on Fig. 1.

10. Creating a keyword map based on abstracts. This step corresponds to the keyword mapping activity. It is described in detail in Section 5.3. The input of this step is abstracts of the secondary set of papers and the fuzzy keyword thesaurus specially developed for this review (see Section 5.3). The output of this step is the keyword map (see Fig. 5).

11. Performing analysis of the keywords map. This step covers analysis of the keyword map. For more details, see Section 5.3. The input of this step is the keyword map. The output is the main trends (see Section 5.3). The main results of this step are presented in Section 5 . 


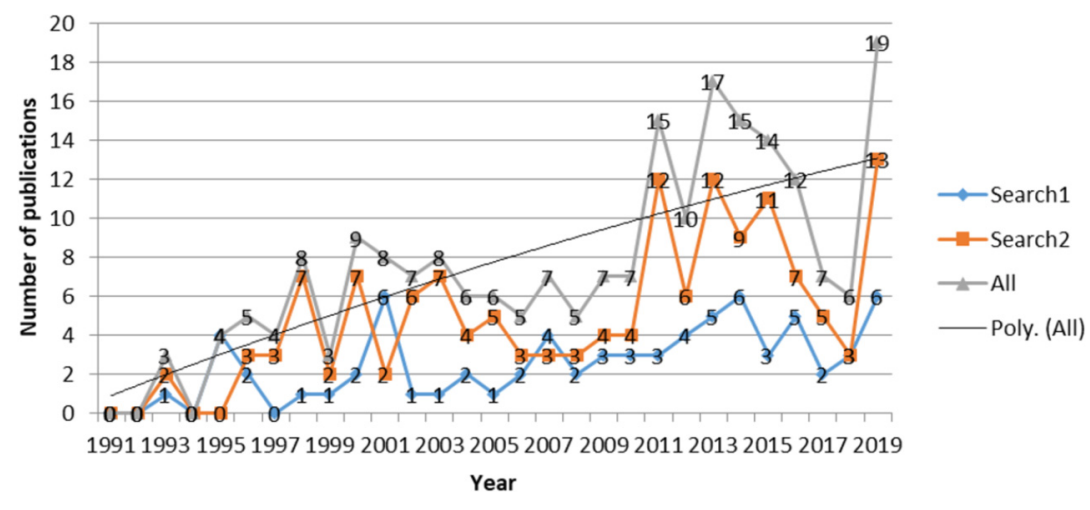

Fig. 3. Number of obtained papers (Poly. (All) - is a trend line).

12. Synchronizing results. In this step, we are synchronizing the obtained results and developing the complexity issues framework (see Section 6).

13. Drawing conclusions. Here, the final conclusions and discussion regarding RQs are drawn.

In Fig. 3, the trend of the research on the topic is illustrated. The number of papers on FIS complexity issues has risen in the period during 2011-2019.

Source evaluation. Authors of Gusenbauer and Haddaway (2020) have compared 28 widely used academic search systems and found that only 14 of 28 are well-suited to SLR, since they met all necessary performance requirements. Among those 14 systems, emphasizing the Computer Science research area, the principal search systems are the following: ACM Digital Library, Bielefeld Academic Search Engine (BASE), ScienceDirect, Scopus, WoS and Wiley Online Library. For this review, we have compared these search systems according to the following criteria: overlapping, scope, quality of the presented research and possibility of full download (not separate download) of search results for bibliometric analysis.

BASE is useful for users without access to paywalled content (Gusenbauer, 2019), which means that large portions of the academic web are not represented. The publisher Elsevier owns both ScienceDirect and Scopus, but Scopus provides possibility to writing more sophisticated strings than ScienceDirect. According to Martín-Martín et al. (2018), WoS and Scopus databases are not overlapping only 12,2\% of documents in Engineering and Computer Science. ACM Digital Library, BASE and Wiley Online Library, having a large number of proceedings publications, lose their advantage because of our predefined EC2, EC3 and EC4 (Dybå and Dingsøyr, 2008). For ensuring quality of publications, databases use different types of impact factors as the following: WoS has an Impact Factor (IF), Scopus has its own CiteScore, which is an alternative to WoS IF. ACM Digital Library and Wiley Online Library count only total citations for each publication. The analysis of the possibility for full download of search results shows that WoS and Scopus have the most carefully arranged bibliographic data. WoS allows downloading up to 500 
items per time, Scopus - full downloading. Summing up all advantages and disadvantages and taking into consideration time and performance constraints, WoS was chosen for this review.

Threats to validity. Here, we discuss the potential threats to validity of this review together with their mitigation actions we have taken. Construct validity refers to the concepts being studied. When defining the review scope and keywords, we faced uncertainty about whether researchers refer to the FIS complexity issues or usage of FIS to solve particular problem domain issues. Consequently, the primary analysis of papers was done to familiarize with the FIS complexity issues and define the related keywords more precisely. Some of the main related works are presented in Section 3. We have used WoS for the search since it enables us to find the most suitable, complete, and not duplicate high-quality refereed papers. For dealing with validity threats regarding the search string (i.e. missing keywords leading to the exclusion of relevant papers), we carried out the primary study during preparation (Miliauskaite and Kalibatiene, 2020a). Moreover, after performing a first iteration of the search, we have applied the backward snowballing technique to develop a new search string from the already included papers. As a result, we have obtained search string 2 for the next iteration of the search. Finally, considering the significant number of the primary set of papers (1380), we have decided that our results and findings are valuable for providing researchers and practitioners with an overview of the state of the art of FIS complexity issues. An internal threat to validity in this research refers mainly to the individual researcher's bias in 1) deciding whether to include or exclude a paper into the secondary set, 2) classifying it according to the complexity issues, and 3) analysing the results. We have used a clearly defined searching strategy, assessed the obtained results independently, and combined the results to minimize the researcher's bias. External validity refers to this review's results and conclusions. They are only valid for the FIS, whose understanding is described in Section 2. We have made great efforts to systematically set up the review protocol and apply it to ensure those general conclusions are valid irrespective of the lack of consensus.

Used tools. Various researchers, like (Li et al., 2017; Chen, 2018; Chen et al., 2019; Vilutiene et al., 2019), have used different science mapping tools, including VOSviewer, BibExcel, CiteSpace, CoPalRed, Sci2, VantagePoint, and Gephi, for analysing, mapping, and visualization of bibliographic data. A detailed review of visualization tools is not the main aim of this paper. We used VOSviewer ${ }^{1}$ as an analysis tool. VOSviewer generates a network from the given bibliographic data. All networks consist of nodes and links. Nodes present documents (i.e. articles), sources (i.e. journals), authors, organizations, countries, or keywords. Nodes with a higher number of occurrences are bigger. Links present relationships among nodes. Thicker links present closer relationships among nodes. Closely related nodes are combined into clusters using a smart local moving algorithm presented in Waltman and Van Eck (2013).

\footnotetext{
${ }^{1}$ https://www.vosviewer.com/.
} 


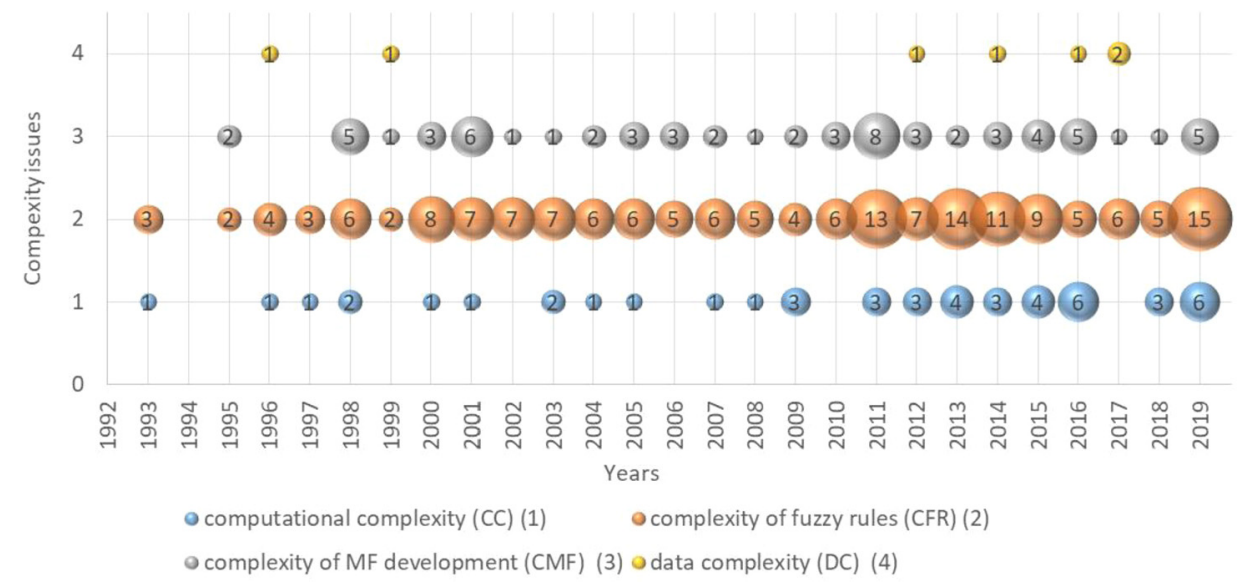

Fig. 4. Found complexity issues according to years.

\section{Results}

\subsection{Found Complexity Issues in FIS (RQ1)}

According to the in-depth content analysis of the abstracts of the secondary set of papers presented in Annex $3^{2}$, four main FIS development complexity issues are found (RQ1) as the following: computational complexity (CC) (1) (i.e. the huge number of calculations in all FIS components); complexity of fuzzy rules (CFR) (2) (i.e. extraction of fuzzy rules, modification and optimization of fuzzy RB, fuzzy RB interpretability); complexity of MF development (CMF) (3) (i.e. MF development, optimization, simplification, partition integrity, comparison of fuzzy numbers, determining MF shape, definition of FOU, aggregation/approximation of information represented by MF); and data complexity (DC) (4) (i.e. related to big data and high-dimensional data issues, balancing of a data set, incomplete input data).

The temporal distribution of the four complexity issues (CC (1), CFR (2), CMF (3), DC (4)) is given in Fig. 4 as follows: $x$-axis presents years, $y$-axis presents four complexity issues, and bubbles indicate the number of papers dealing with a particular complexity issue in a given year. The larger the bubble, the more significant number of papers dealing with a specific complexity issue in a given year.

As can be found from Fig. 4, the computational complexity (CC) issue originated in 1993. CC of each method may be different. It is commonly referred to as time complexity (Banaeian et al., 2018). Authors of Aghaeipoor and Javidi (2019) evaluate the average running time for each stage of MOKBL (Multi-Objective Knowledge Base Learning through a fuzzy feature selection and slight tuning of MFs) + MOMs (Multi-Objective Modifications through a rule pruning process and main tuning of MFs). The running times are

\footnotetext{
${ }^{2}$ https://github.com/Jolantux13/repository.
} 
different for different datasets. The authors said that their proposed method was not timeconsuming since they applied efficient strategies for rule generation, objective estimation, new rule pruning, and tuning. The relatively short running time demonstrates the model's efficiency and scalability, especially for the most complicated datasets (Aghaeipoor and Javidi, 2019). Authors of Cavalieri and Russo (1998) mention computation complexity together with acceptable memory resource requirements. In Hata et al. (2016), authors analyse tuning of fuzzy rules using a neuro-fuzzy learning algorithm. However, as they state, increasing the number of inputs greatly increases the number of parameters. Thus, learning time increases and learning accuracy decreases. Authors of Ruiz-Garcia et al. (2019) consider the practical implementation of general type-2 fuzzy logic systems (GT2-FLSs) and state that the type-reduction operation still presents a challenge for GT2-FLSs because of its CC. They introduce new equations for the meet and join operations, type reduction procedure for the type-2 fuzzy logic system, fuzzification, inference, type-reduction, and defuzzification to reduce CC. Summing up, the CC issue is closely related to other identified complexity issues. It remains relevant from the beginning of the development and application of fuzzy set theory until now; since fuzzy set theory application involves many computations because of its complexity.

The second row in Fig. 4 presents the complexity of fuzzy rules (CFR). As can be mentioned, it is the most significant and relevant issue among others. As stated by some authors, like (Marimuthu et al., 2016; Rajeswari and Deisy, 2019; Hilletofth et al., 2019), the optimal number of the premise and consequent parameters along with the reduced number of rules should be made to achieve more accuracy and reduce the computational complexity by triggering a smaller number of nodes for each activity in fuzzy logic systems (FLS). The significant issues that affect the accuracy of fuzzy-based methods are fixing appropriate MF and validating the fuzzy rules before extracting outliers (Rajeswari and Deisy, 2019). Authors of Hilletofth et al. (2019) investigate the possibility of increasing the interpretability of fuzzy rules and reducing the complexity when designing fuzzy rules. They advocate using of three novel fuzzy logic concepts (i.e. relative linguistic labels, high-level rules and linguistic variable weights) in a fuzzy logic system for re-shoring decision-making and, consequently, increasing the interpretability of fuzzy rules and reducing the complexity when designing fuzzy rules while still providing accurate results.

An essential issue in the design of fuzzy rule-based classification is the automatic generation of fuzzy if-then rules and MFs (Ravi and Khare, 2018), i.e. choosing the type of MFs, the number of MFs, and defining the parameters of MFs. Authors propose a brain genetic fuzzy system (BGFS) for data classification by newly devising the exponential genetic brain storm optimization, i.e. MFs and rules are derived using the exponential brain storm optimization algorithm. So, CFR stands together with other complexity issues, especially the complexity of MF development (CMF). It remains relevant from the beginning of the development and application of fuzzy set theory until now. Its slight increase is visible in 2011-2019.

The third row in Fig. 4 presents the historical evolution of the CMF issue. As was mentioned before, it concerns choosing the type of MFs, the number of MFs or partitioning, defining parameters of MFs, the integrity of partitions (Aghaeipoor and Javidi, 2019; 


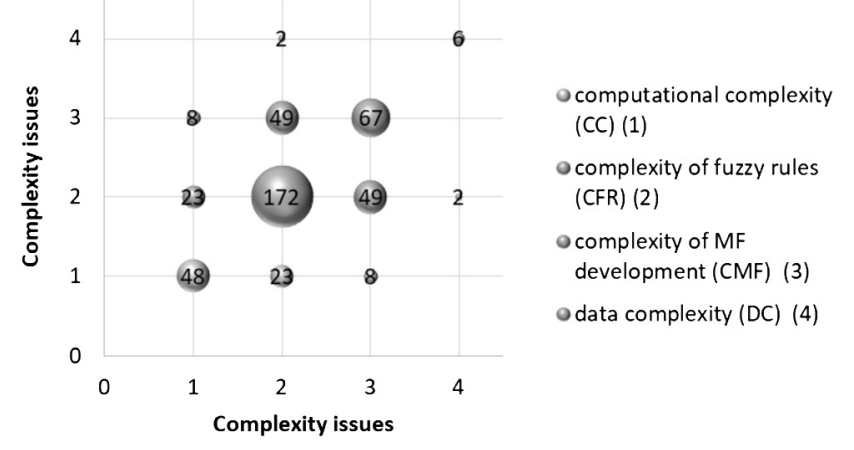

Fig. 5. Co-relation of the found complexity issues.

GaneshKumar et al., 2014), and comparison of fuzzy numbers (Matarazzo and Munda, 2001). As the selected papers' analysis shows, this issue is analysed in tandem with complexity issue of fuzzy rules, like in Rajeswari and Deisy (2019), Ravi and Khare (2018), Barsacchi et al. (2019).

The fourth row in Fig. 4 presents the data complexity (DC) issue. Its occurrence in this research is weak. This limitation can be explained by the fact that the fuzzy set theory is mostly used to solve DC, like high dimensional or spatial data, noisy data, and big data (Chang et al., 2016; Chan et al., 2018; Wang et al., 2017; Feng et al., 2019), but not viewed as a complexity issue in FIS development.

Summing up, those complexity issues found are a relevant research area from the beginning of the development and application of fuzzy set theory until now. Moreover, they evolve with the origin of new technologies and approaches, like neural networks and genetic algorithms, to optimize fuzzy rules and MFs. Additionally, they correlate with each other. Therefore, they should be analysed in tandem.

\subsection{Co-Relationship of Complexity Issues}

Figure 5 presents a co-relationship analysis between different pairs of complexity issues.

The $x$-axis and $y$-axis present the same four complexity issues in the figure, and bubbles indicate the number of papers dealing with a pair of complexity issues. The larger the bubble, the more significant number of papers dealing with a pair of complexity issues. Moreover, Fig. 5 is symmetric since the logical AND of two sets provide symmetric results.

As can be seen in the figure, the most related complexity issues are CFR and CMF (analysed in 49 papers), the second relevant - CC and CFR (in 23 papers), the third relevant $-\mathrm{CC}$ and $\mathrm{CMF}$ (in 8 papers), and the least relevant - CFR and DC (in 2 papers). CC and DC, CMF, and DC are not found to be related. A co-relationship analysis among three complexity issues shows that the most related triplet is $\mathrm{CC}-\mathrm{CFR}-\mathrm{CMF}$ (in 5 papers). A corelationship analysis among the found complexity issues indicates that no papers analyse all four issues in co-relationship. 


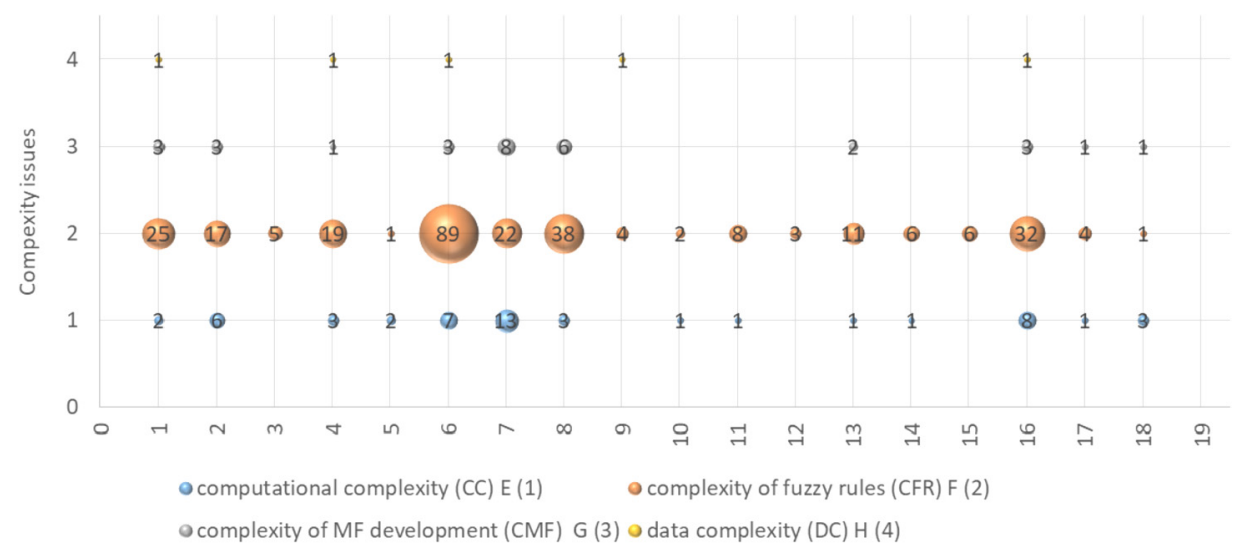

Fig. 6. Found solutions for complexity issues (x axis: 1 - genetic algorithm, 2 - neural network, 3 - particle swarm, 4 - other evolutionary algorithm, 5 - MFs type reduction, 6 - fuzzy rule reduction, 7 - MFs reduction, 8 - optimization, 9 - decision table/tree, 10 - granularity, 11 - mining, 12 - Pareto, 13 - similarity, 14 - least square, 15 - fitness function, 16 - approximation techniques, 17 - aggregation, 18 - fuzzy c-means, 19 - other).

Summing up, it is found that one particular complexity issue is analysed in 147 papers from 217 selected papers $(67.74 \%)$, two complexity issues in tandem are analysed in 64 papers $(29.49 \%)$, three - in 6 papers $(2.76 \%)$, and four - not found. This comparison allows us to conclude that authors tend to analyse one particular issue, less prone to two related issues, and do not analyse all the issues in conjunction.

\subsection{Found Solutions for Complexity Issues (RQ2)}

Fig. 6 depicts found solutions for complexity issues in FIS. The detailed primary information is presented in Annex $1^{2}$. The 19 practical solutions have been found in the analysed papers (RQ2). They are as follows: 1) genetic algorithm, 2) neural network, 3) particle swarm, 4) other evolutionary algorithms, 5) MFs type reduction, 6) fuzzy rule reduction, 7) MFs reduction, 8) optimization, 9) decision table/tree, 10) granularity, 11) mining, 12) Pareto, 13) similarity, 14) least square, 15) fitness function, 16) approximation techniques, 17) aggregation, 18) fuzzy c-means, and 19) other. As can be seen, some solutions are general, like optimization (Guély et al., 1999) or reduction (Lin et al., 2015); others, on the contrary, are specific techniques, like fuzzy c-means (Fu et al., 2019), Pareto (Alcalá et $a l ., 2009 b$ ), etc. Therefore, they should be systematized into a hierarchy. To understand the complexity issues found and possible solutions, they are analysed and organized further in Section 6.

The CC issue (Fig. 6) is solved by: MFs reduction (7) (Lee et al., 2011; Akbarzadeh-T et al., 2000) (i.e. when there are less MFs, there are fewer computations), using different approximation techniques (16) (i.e. the techniques are used to interpolate and simplify MFs in inference/reasoning process; consequently $\mathrm{CC}$ is reduced), fuzzy rule reduction (6) (Lin et al., 2015; Ang and Quek, 2005; Tikk and Baranyi, 2000; Kóczy and Hirota, 1993) (i.e. when there are less fuzzy rules, the inference/reasoning process is simpler; 
consequently we need fewer computation resources), neural networks (2) (i.e. neural networks are used to achieve dynamic rule formation in FIS (Marimuthu et al., 2016; Lin et al., 2015; Ang and Quek, 2005; Kóczy and Hirota, 1993), thus reducing CC), etc.

The CFR issue (Fig. 6) can be mitigated by the following means: 1) reducing the number of fuzzy rules (6) (Lin et al., 2015; Ang and Quek, 2005; Tikk and Baranyi, 2000; Kóczy and Hirota, 1993), 2) optimizing the number of fuzzy rules (8) by choosing only appropriate (Bouchachia and Vanaret, 2014; Renhou and Yi, 1996), 3) applying a particular approximation technique (Rojas et al., 2000); 4) using genetic algorithms (Akbarzadeh-T et al., 2000; Balazs and Koczy, 2012; Ishibuchi and Yamamoto, 2003); 5) MFs reduction (Akbarzadeh-T et al., 2000), etc.

The CMF issue (see Fig. 6) can be resolved by the following means: 1) reducing MFs (7) (Lee et al., 2011; Akbarzadeh-T et al., 2000), 2) optimization (8) (Akbarzadeh-T et al., 2000), 3) neural network (2) (Lee et al., 2011; Galende-Hernández et al., 2012), etc. The data complexity is reduced using genetic algorithms (1), other evolutionary algorithms (4), fuzzy rule reduction (6), approximation techniques (16) (for explanations, see below in this section) and decision table/tree (9) (Antonelli et al., 2016).

As can be seen from the analysis of the found solutions for complexity issues, some issues are solved through minimizing others (like the computational complexity is minimized by reducing rules, etc.). Therefore, the synergy effect between complexity issues is observed, like the positive synergy between rule selection and tuning techniques to enhance the capability to obtain more accurate and compact fuzzy logic controllers (Alcalá et al., 2009a). The synergy among application of several techniques leads to a positive result, like successful scoping with large and high-dimensional data sets (Antonelli et al., 2013), a positive performance is achieved when applying genetic tuning after the application of the proposed method (Sanz et al., 2012); fuzzy c-means clustering and granularity with weighted data is implemented for enhancing the performance of the classifier (Fu et al., 2019).

In Fig. 7, the distribution of applied solutions for complexity issues is presented by years. As can be seen, genetic algorithms (1) are used in conjunction with fuzzy logic theory continuously throughout the analysed years. According to Lee et al. (2014), a genetic fuzzy system hybridizes the FIS approximate reasoning with the learning capabilities of evolutionary algorithms to improve the overall behaviour of the resulting system. Authors of Stavrakoudis et al. (2012), Sanz et al. (2011), Casillas et al. (2005), Kim et al. (2006b) have used genetic tuning to improve the performance of fuzzy rule-based classification systems. In Rey et al. (2012), relevant rules were selected using a genetic algorithm that took into account the information obtained by an orthogonal transformation.

Neural networks (2) are integrated into FIS to enable effective handling of the fast and rapidly changing data streams (Ferdaus et al., 2019). The author Chen (2015) has proposed a neuro-fuzzy approach with a learning strategy, which allows us to add or prune fuzzy rules and allocate suitable positions of MFs to perform subsequent optimization efficiently. Authors of Farag et al. (1998) have used a fuzzy neural network to find initial MFs of the fuzzy model and to extract fuzzy rules, and a genetic algorithm to optimize tuning of the found initial MFs. 


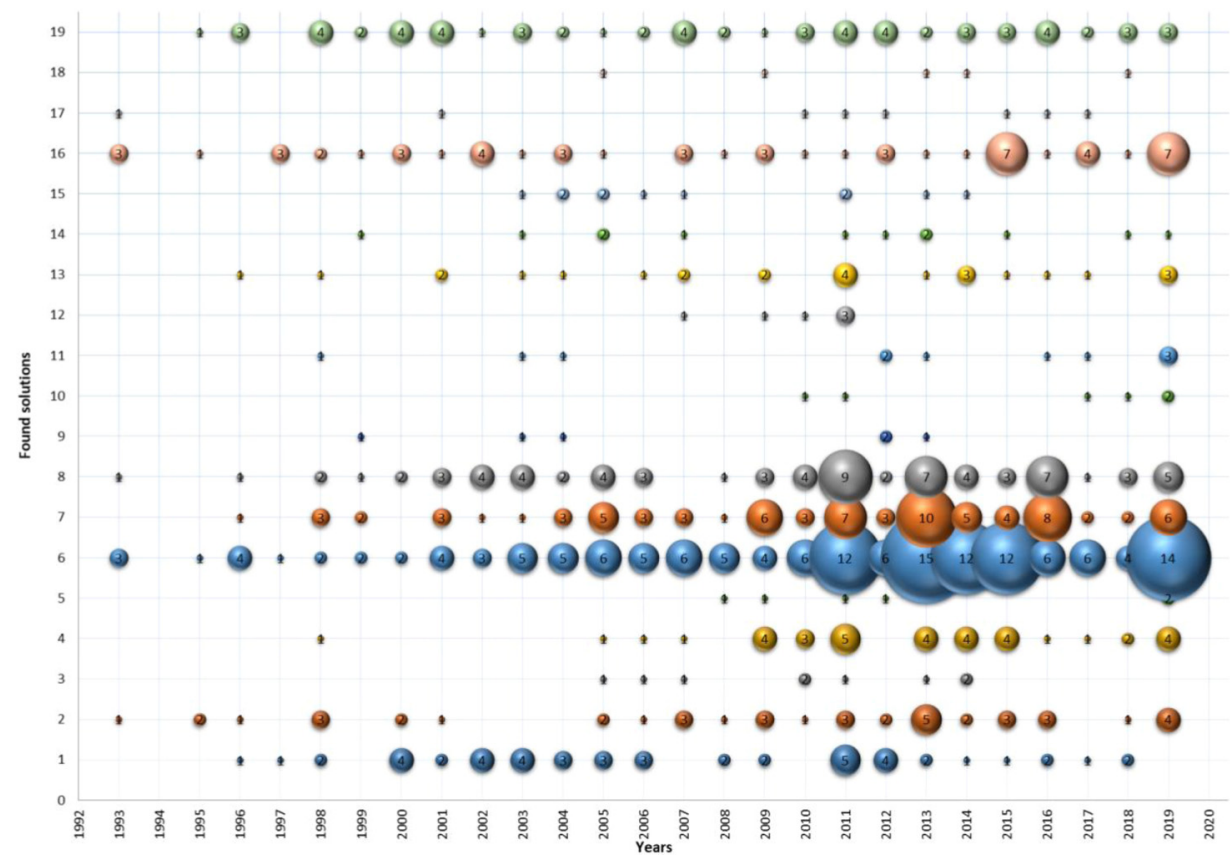

Fig. 7. Distribution of applied solutions for complexity issues according to years ( $y$ axis: ( $y$ axis: $1-$ genetic algorithm, 2 - neural network, 3 - particle swarm, 4 - other evolutionary algorithm, 5 - MFs type reduction, 6 - fuzzy rule reduction, 7 - MFs reduction, 8 - optimization, 9 - decision table/tree, 10 - granularity, 11 mining, 12 - Pareto, 13 - similarity, 14 - least square, 15 - fitness function, 16 - approximation techniques, 17 aggregation, 18 - fuzzy c-means, 19 - other).

Since 2009, some evolutionary algorithms (4) have been used to solve different complexity issues. Authors have not mentioned the names of evolutionary algorithms in their abstracts but have just said that they used evolutionary algorithms in their research. We have distinguished them from other approaches, but there is a high probability that most of them are neural networks or genetic algorithms.

Since 2005, a particle swarm (3) optimization have been employed to manage the clustering task for a complex, irregular, and high dimensional data set (Feng et al., 2006); to simultaneously tune the shape of MFs and the rule consequences for the entire fuzzy rule base (Chatterjee and Siarry, 2007); to explore the fuzzy rule sets, fuzzy sets and MFs to its optimal or the approximately optimal extent (Huang et al., 2010); to optimize the parameters (i.e. premise (antecedent) parameters, consequent parameters and structure of fuzzy rules) of the proposed fuzzy classifiers (Elragal, 2014).

MFs type reduction (5) becomes popular since 2008. It can be explained by the more active usage of type-2 fuzzy sets, which are more complex and require more computational efforts. Consequently, different MFs type reduction procedures are proposed, like in RuizGarcia et al. (2019), Lee et al. (2011), Shahparast and Mansoori (2019).

Fuzzy rule reduction (6) and MFs reduction (7) are the most popular complexity issue solving approaches. They encompass evolutionary lateral tuning of MFs and fuzzy rules 
in Alcalá et al. (2007), eliminating some of the rules by combining different kinds of reference inputs (Kondratenko et al., 2013), introducing a set of governing equations for designing MFs and fuzzy rules to preserve the monotonicity property in Jee et al. (2013), using the Hebbian learning mechanism for rule pruning in Nguyen et al. (2015).

Different optimization (8) procedures are used widely for solving complexity issues as well. In Marimuthu et al. (2016), authors have proposed an optimized adaptive neurofuzzy inference system (ANFIS) using frequent pattern mining (see also mining (11) in Fig. 7) and FIS for recognition of activities of a person. Authors make initial rule identification through MFs of each activity, and the number of rules is reduced using the frequent pattern mining (FPM) approach. During the learning phase, the optimal premise parameters are selected using the gradient descent method (other (19) in Fig. 7), and the choice of consequent parameters is based on the least-square (least square (14) in Fig. 7) estimation method. In Ravi and Khare (2018), MFs and rules are devised using an exponential genetic brainstorm optimization algorithm. Authors of Altilio et al. (2018) have used the Adaptive Neuro-FIS to optimize the generalization capability of the resulting model, i.e. to estimate numerical parameters of each fuzzy rule (i.e. MFs) and the whole number of rules to be used. In Almasi and Rouhani (2016), authors have proposed a method based on optimization to generate appropriate MFs and solve classification problems simultaneously. MFs are built based on dynamic class centres.

Decision tables/trees (9) are not so popular for solving complexity issues. Several authors have used them to derive fuzzy if-then rules and MFs from a set of given training examples (Hong and Chen, 1999) or for generating an initial rule base (Barsacchi et al., 2019). Since 2010, a granularity approach (10) has been used for fuzzy partition granularity (Barsacchi et al., 2019; Ishibuchi et al., 2011) and information granularity (Zhu et al., 2017; Fu et al., 2019). Mining techniques (11) have been weakly used for solving FIS development issues, except pattern mining for the reduction of rules in Marimuthu et al. (2016), fuzzy rule mining in Aghaeipoor and Eftekhari (2019), Olufunke et al. (2013), fuzzy association rule mining in Antonelli et al. (2016). Since 2008, Pareto optimization (12) has been used in a few studies, like (Alcalá et al., 2009b; Ishibuchi et al., 2011; Gacto et al., 2010).

Similarity measure (13) is continuously used in some studies for the following goals: for cluster merging utilizing both similarities of the adapted clusters and their centre closeness (Alaei et al., 2013), for partition integrity measure by using an index based on the similarity between the partitions (Antonelli et al., 2011), to alleviate overlap among MFs and to reduce the complexity of the obtained system (Leng et al., 2009), to combine similar input linguistic term nodes for fuzzy sets that indicate the degree to which two fuzzy sets are equal (Chao et al., 1996), for measuring similarity between two fuzzy terms, by their closeness derived from their distance (Kóczy and Hirota, 1993).

Some studies have used the least square method (14). In Pratama et al. (2013), the optimal fuzzy consequent parameters are updated by the time localized least square method. An orthogonal least squares method and a total least squares method are used for rule selection in nonlinear plant modelling problems (Yen and Wang, 1999). In Wang et al. (2005), a recursive least square together with fitness function (15) (Fig. 7) is used to obtain 
the optimized fuzzy models. The fuzzy rule parameters of antecedents (i.e. MFs) are randomly generated, and the rule consequents are estimated using a regularized least-squares algorithm (Altilio et al., 2018).

A fitness function (15) has been found among other rarely used methods for solving different complexity issues, like to reduce the number of rules and to maintain the performance of FIS (Pal et al., 2003), optimize parameters of antecedent MFs and rule structures (Kim et al., 2006a). Authors of Kim et al. (2005) have used a new fitness function to optimize a fuzzy model, i.e. to obtain modelling accuracy, rule compactness, and interpretability of input MFs. Approximation techniques (16) were used for fuzzy mapping (Tikk and Baranyi, 2000; Kóczy and Hirota, 1993; Rojas et al., 2000), approximating the secondary MF (Melin et al., 2019), and approximating reasoning characteristics of FIS (Golestaneh et al., 2018). Aggregation (17) is found rarely, like (Gegov et al., 2015; Mesiarová-Zemánková and Ahmad, 2012; Eslamkhah and Hosseini Seno, 2019). Fuzzy c-means (18) have been used for process fault detection and diagnosis (Alaei et al., 2013), solving data clustering problems (Ramathilaga et al., 2014), generating fuzzy rules (Emami et al., 1998), forming an initial fuzzy model (Fu et al., 2019). Other (19) approaches were found in the analysed papers only once. Some of them are discussed previously in this section. They are not mentioned again to avoid duplication.

Summing up, from Fig. 7, we can conclude that artificial intelligence approaches (i.e. genetic algorithms, neural networks, other evolutionary algorithms), simplification and generalization techniques (i.e. reduction, similarity, etc.), optimization techniques (i.e. Pareto), similarity techniques (i.e. fitness function, least square) are used for solving different complexity issues in FIS development.

\subsection{Keyword Map}

In this section, we provide the keyword co-occurrence analysis to present the main content of the selected papers and the range of researched areas in complexity issues. The cooccurrence analysis of the keyword map is performed using the authors' abstracts.

VOSviewer selects keywords using an automatic keyword identification technique (van Eck et al., 2010) and creates the keyword map by considering the closeness and strength of existing links. The closeness and strength are calculated from the number of papers, in which both keywords have occurred together (i.e. using binary counting). VOSviewer also clusters keywords and portrays the topic by colours. The size of the bubbles presents the density of occurrence of keywords. VOSviewer uses a unified approach to keywords' mapping and clustering (Khalil and Gotway Crawford, 2015). In this research, it has identified 4566 keywords, occurring at least 7 times in the abstracts of 217 papers, and develop the keyword map (Fig. 8). A fuzzy keyword thesaurus was created to perform data cleaning as follows:

1. merge different spellings of the same word, like "membership function" and "membership functions";

2. merge abbreviations with full keywords, like "membership functions", "MFs" and "MF"; 


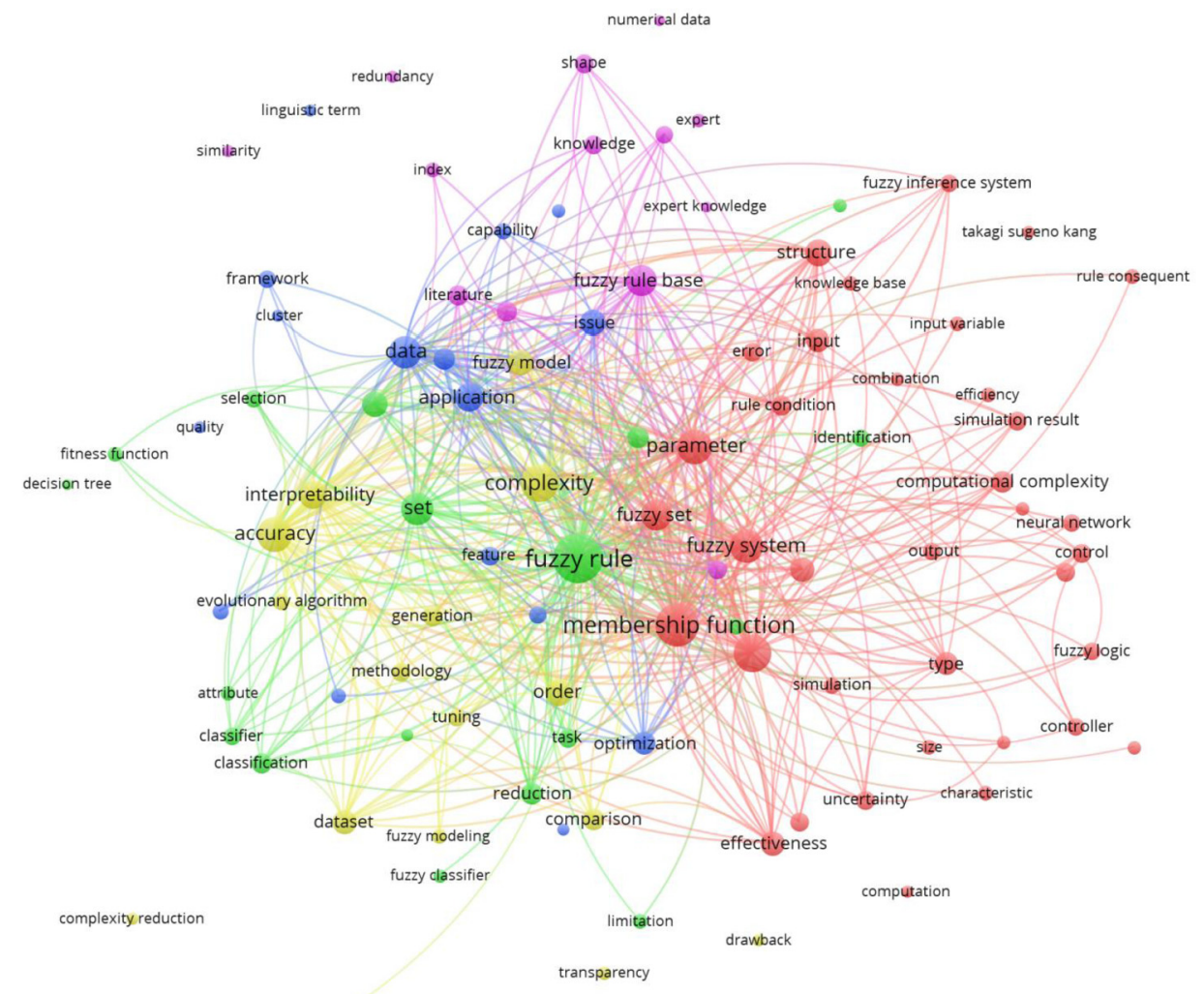

classification system

Fig. 8. Co-occurrence network of keywords on the topic of complexity issues.

3. merge synonyms, like "rule premise" and "rule condition";

4. exclude general keywords, like article, aim, paper, etc., since these keywords provide very little information, and the usefulness of a map tends to increase when they are excluded. ${ }^{3}$

The thesaurus consists of 226 merged and 215 excluded keywords (see Annex $2^{2}$ ).

Finally, 100 keywords that seemed most relevant and most interesting were presented in the keyword map (Fig. 8) and used for in-depth analysis. We have analysed the keyword map based on the complexity issues that are named as complexity (78 occurrences found in the abstracts), issue (37), computational complexity (28), limitation (14), drawback (8) and redundancy (8). The most relevant co-occurred complexity issue keywords are the following:

\footnotetext{
${ }^{3}$ https://www.vosviewer.com/documentation/Manual_VOSviewer_1.6.8.pdf.
} 


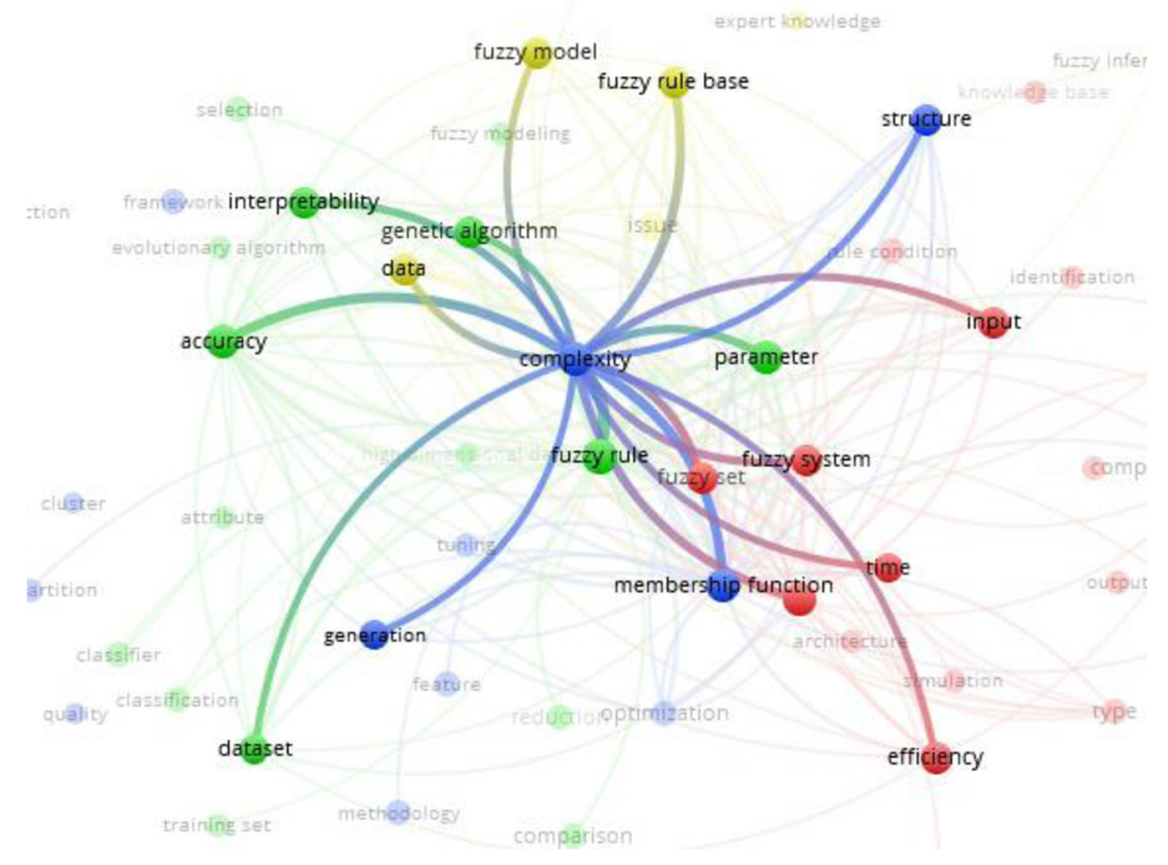

Fig. 9. Co-occurrence of the keyword "issue".

1. Complexity - fuzzy rule, fuzzy rule base, membership function, accuracy, performance, fuzzy set (their co-occurrence strength is $>24)^{4}$ (Fig. 9). Accuracy maximization and complexity minimization are two important goals of FIS (GaneshKumar et al., 2014). In Soua et al. (2013), the authors propose reducing the complexity by decreasing the number of rules and antecedent conditions in solving pattern classification problems. The authors of Bouchachia and Vanaret (2014) propose to maintain low complexity of a rule base using online optimization and feature selection mechanisms. The authors of Juang and Juang (2012) propose to reduce the number of interval type-2 fuzzy sets by merging them and, thus, improve complexity-based interpretability.

2. Issue - fuzzy rule, membership function, parameter, accuracy, fuzzy system, data and dataset (their co-occurrence strength is $>12)^{4}$ (Fig. 10a). The analysis of abstracts shows that major issues that affect the accuracy of FIS are fixing appropriate MFs and validating fuzzy rules before inferencing, like extracting outliers in (Rajeswari and Deisy, 2019), classification in Ravi and Khare (2018), Almasi and Rouhani (2016), inferencing in Altilio et al. (2018), etc. The main issues related to data and datasets are noise (Ramathilaga et al., 2014) and imbalance (Chaudhuri, 2014).

3. Limitation - fuzzy rule, membership function, parameter, fuzzy set, performance (their co-occurrence strength is $>5)^{4}$ (Fig. 10b). Computational cost represents a limitation

\footnotetext{
${ }^{4}$ Note that for each complexity issue keyword co-occurrence strength differs. The same cannot be chosen because of different occurrence of complexity issue keywords, which are shown in the brackets.
} 


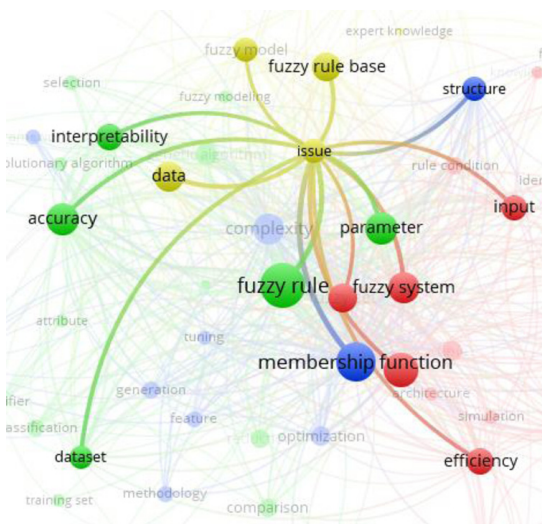

(a)

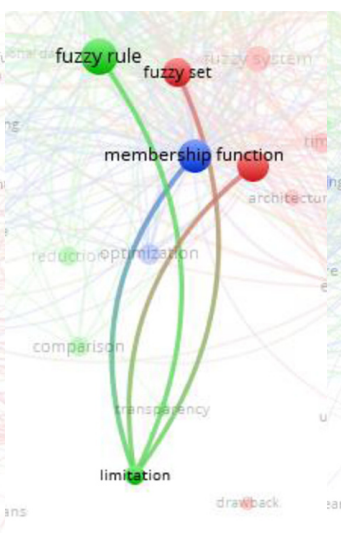

(b)

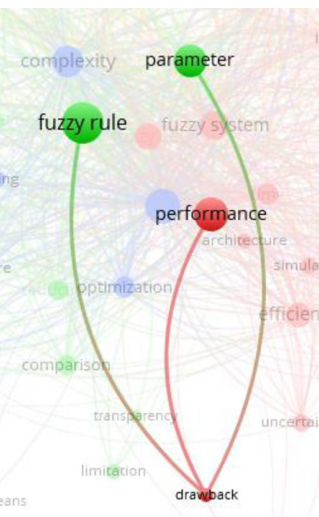

(c)

Fig. 10. (a) Co-occurrence of the keyword "issue". (b) Co-occurrence of the keyword "limitation". (c) Cooccurrence of the keyword "drawback".

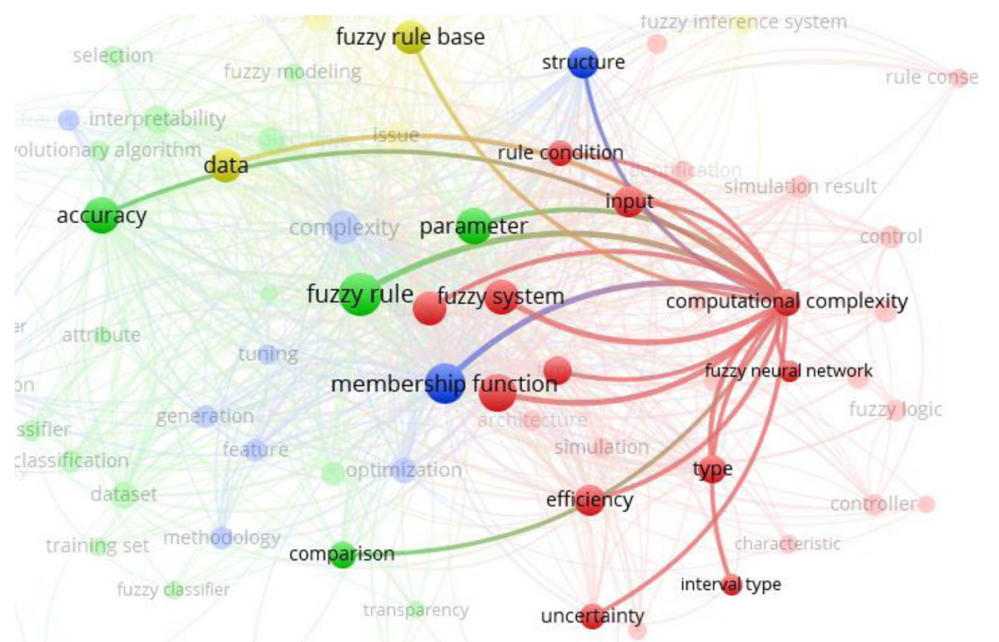

Fig. 11. Co-occurrence of the keyword "computational complexity".

for many FIS (Melin et al., 2019). The authors of Di et al. (2001) name the limitations of a fixed MF. Pawlak's rough MFs have limitations when handling incomplete data that exist widely in the real world (Ge et al., 2017). The authors of Fu et al. (2019) proposed constructing a fuzzy classifier using justifiable granularity with weighted data to alleviate limitations and compromise between the accuracy and the number of rules in the classifier.

4. Drawback - fuzzy rule, parameter, performance (their co-occurrence strength is $>3)^{4}$ (Fig. 10c). In Zhao et al. (2015), the main drawback of FIS is the model training process for extracting a sparse set of fuzzy rules. 
5. Computational complexity - fuzzy rule, membership function, performance, fuzzy system, parameter, type (their co-occurrence strength is $>9)^{4}$ (Fig. 11). In Lee (2019), Ruiz-Garcia et al. (2019), the type-reduction operation still presents a challenge in FIS because of its CC. In Lee and Pan (2009), the authors proposed using interval-valued neural fuzzy systems with asymmetric MFs to enhance the performance and reduce CC. The problem of sparse fuzzy rule bases is the high CC of inference method (Koczy and Hirota, 1997).

Summing up, the following main complexity issues can be highlighted: computational complexity, complexity of fuzzy rules, complexity of MFs, and data complexity, which are the same as obtained during SLR (Table 3).

\section{Framework of Complexity Issues and Their Possible Solutions in FIS Development}

In this section, we present a framework of complexity issues and their possible solutions in FIS development. The developed framework serves as a map for identifying, understanding and solving complexity issues in FIS development. It (Fig. 12) consists of two following dimensions: Horizontal dimension - presents co-relationship dependencies among complexity issues of FIS development, based on Fig. 5. Those complexity issues co-relate in tandem, i.e. the occurrence of one complexity issue leads to the rise of another. Vertical dimension - presents a holistic view of complexity issues of FIS development. It begins with the general FIS development complexity issue, which has the highest granularity. Applying the top-down approach, which encapsulates the principle of decomposition and abstraction, we move down by refining and granulating the general FIS development complexity issue to small problems, for which solutions are known or can be defined.

Based on the obtained results and FIS reference architecture (see Fig. 1), all found complexity issues can be classified as presented in Fig. 12. They are the following:

1. Computational complexity refers mainly to Computational Time and Other Computational Resources (memory, space, etc.) required for fuzzification, inference and defuzzification.

2. Data complexity. It refers to the complexity issues related to data that are necessary for automatic generation of MFs and fuzzy rules. The data can be noisy, incomplete, high-dimensional or big data. This Data Complexity issue can be viewed from the lens of Big data properties (Wang et al. (2017); Firican (2017)) that are the following: value, velocity, volume, variety, variability, validity, veracity, vulnerability, volatility and visualization.

3. Knowledge complexity. It refers to MFs development complexity and Fuzzy rule complexity. Those two complexities are joined into Knowledge complexity, since they are tightly related and depend on each other. Moreover, they are obtained from the application domain data.

Solutions found in the analysed papers are listed below the identified complexity issues (see Fig. 12). Those solutions are explained in Section 5.3. A number near each 


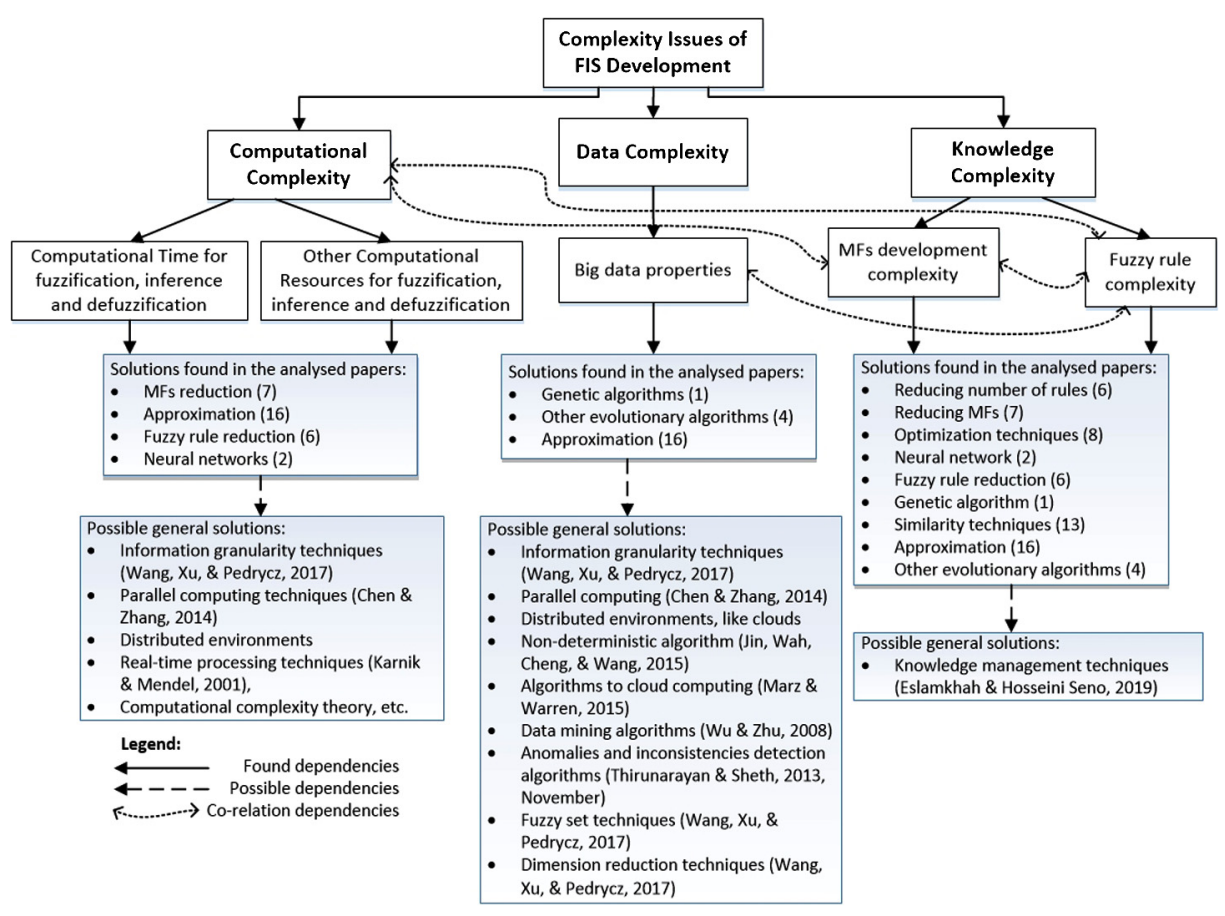

Fig. 12. The framework of complexity issues and their possible solutions in FIS development.

solution identifies its number in Fig. 6. On a more global scale, going beyond FIS, the identified complexity issues are not new in the Computer Science field. Therefore, based on a worldwide perspective, Possible general solutions are added below solutions found in the analysed papers. They show the directions in which FIS can and should be developed. The detailed analysis of Possible general solutions is not performed here since it is outside the scope of this paper.

\section{Discussion}

As FIS become increasingly complex because of their application domain and tasks being solved, FIS development complexity issues need to be systematized and classified to ensure its development efficiency and effectiveness. In this research, we have applied a hybrid SLR and SMS to answer the defined research questions: (RQ1) What complexity issues exist in the context of developing fuzzy inference systems (FIS)? and (RQ2) Is it possible to systematize existing solutions of identified complexity issues? The conducted review on the topic shows an increase of papers analysing different complexity issues in FIS development. It can be attributed to technological development and raise the applicability of fuzzy theory to present uncertainties in various application domains.

Finally, we can summarize the obtained results and answer the research questions. Four main issues have been found in the reviewed papers (RQ1): 1) computational complexity 
(CC), 2) complexity of fuzzy rules (CFR), 3) complexity of MF development (CMF), and 4) data complexity (DC). These complexity issues did not occur with equal frequency in the analysed papers as the following: CFR had the highest occurrence, CMF - high occurrence, $\mathrm{CC}$ - moderate occurrence, and DC - low occurrence. Here, it is necessary to discuss why we have this phenomenon of circumstances. CFR and CMF have occurred mostly, since MFs and fuzzy rules development are central in developing FIS (Fig. 1). The defined MFs and fuzzy rules form a fuzzy model impacting the FIS inferencing results. Thus, the more precise the definition of MFs and fuzzy rules is, the more accurate results and the more efficient FIS we will get. Moreover, we express domain knowledge through MFs and fuzzy rules. Therefore, in our proposed framework of complexity issues and their possible solutions in FIS development, CMF and CFR are generalized as Knowledge Complexity. CC has occurred moderately. It is an important complexity issue in FIS development, since we have to perform numerous calculations in each FIS component. However, in the analysed papers, not all authors mention this complexity issue in their abstracts. They highlight CFR and CMF and believe that their solution enables the reduction of CC. Although solving CFR and CMF does not entirely reduce $\mathrm{CC}$, it depends on calculations in other components of FIS, like defuzzification, but reduces it significantly. Consequently, to increase $\mathrm{CC}$, we need an efficient way of developing all FIS components. DC has the lowest occurrence in the analysed papers. DC is a global issue going beyond FIS. Since we limit our search strings to FIS but not to all software systems, we got a small number of papers highlighting DC. Moreover, as observed, FIS is usually used to address DC, rather than DC being the FIS development complexity issue. Therefore, we need to extend the review with new keywords, like "high-dimensional data," "big data," "noisy data," etc., to analyse DC more accurately. However, it is outside the scope of this review.

We have performed the co-relationship analysis between different pairs, triples, and quadruples to determine the most related complexity issues. One particular complexity issue is most relevant in the analysed papers (found in 217 papers, 67.74\%). Pairs of complexity issues are analysed in 64 papers $(29.49 \%)$, triplets - in 6 papers $(2.76 \%)$, and a quadruple - not found. This comparison allows us to state that authors tend to analyse one particular issue, less often - two or three related issues, and do not analyse all the issues in conjunction. The authors choose the complexity issues that are most relevant to their research and reduce them using a particular approach. Besides, they expect that reducing the most relevant complexity issue will reduce other related complexity issues. Therefore, in the domain for in-depth knowledge elicitation, a deeper analysis of causal relationships among complexity issues is necessary by applying the causality-driven methods (Gudas et al., 2019) and determining fuzzy relations (Ferrera-Cedeño et al., 2019) among complexity issues.

This paper has proposed the framework of complexity issues and their possible solutions in FIS development (RQ2). It allows us to systematize existing solutions for each complexity issue found in RQ1. As can be seen, the same solutions, like genetic algorithms, neural networks, approximation and optimization techniques, etc., are used to solve different complexity issues. From a global perspective, the found complexity issues are not new in CS. Therefore, to solve those complexity issues, we propose to employ well-known 
Possible general solutions (Fig. 12). Moreover, they show the directions in which FIS can and should be developed and refined. Summarizing, complexity issues in FIS development should be solved by searching for new and applying already known approaches from more general fields, like computational complexity theory, parallel computing techniques, information granularity techniques, knowledge management techniques, etc.

One more result of this review is the application of the hybrid SLR and SMS approach. The advantage of the applied approach is that it includes SLR's advantageous properties to perform in-depth analysis for answering the defined research questions and SMS with a keyword map visualizing and better understanding each concept's real meaning in FIS development.

The overall advantage of the results obtained in this paper is that they enhance knowledge on FIS development and help the researchers and practitioners become familiar with the found FIS development complexity issues and their possible solutions.

\subsection{Limitations of the Review}

The most common limitations of systematic reviews are related to coverage of search and to possible biases introduced during study selection, data extraction, and analysis. These are also the main limitations of this review. The coverage of the searching threat is related mainly to the search Source of this review, since for the search, we have chosen only one search engine, i.e. WoS. However, in Section 3, we have argued WoS selection. Moreover, our iterative search allows us to obtain a significant number of the primary set of papers (1380), which is a sufficient size of an initial set to perform the review. Moreover, we concentrate on the papers published in Computer Science, Information Systems, and Software Engineering. Although performing a systematic review of other fields was not the aim of our review, we understand that important papers might have been excluded from our discussions. A systematic review performed manually across all fields may not be feasible. Consequently, we intend to conduct a systematic review focusing on a field related to FIS development and keeping the review process feasible.

We addressed potential research bias in assessing papers, data extraction, and data analysis by strictly following the predefined review protocol, assessing the obtained results independently, and then combining them to minimize the researcher's bias. Two researchers performed all tasks independently and then merged the results.

Finally, since the review process is described in detail, it allows us to ensure the review's replicability. However, there is no guarantee that other researchers will obtain the same results (but similar ones) as presented in this work since subjectivity cannot be eliminated.

\subsection{Lessons Learned}

The lessons learned from this review can be organised according to two factors: those related to the performing of a systematic review and those related to the complexity issues and their possible solutions in FIS development. 
Lessons learned regarding the literature review. There has been an increasing number of contributions related to the analysed topic in recent years. Researchers need to review a greater amount of papers, which may lead to general conclusions and developments in FIS area. However, the big amount of papers, heterogeneity of searching sources (i.e. inconsistent search fields, inconsistent syntax and filters, inconsistencies in exportable formats, limited number of exportable citations, inconsistencies in exportable data, etc. (Shakeel et $a l ., 2018$ ) and manual review process makes it difficult to conduct more general reviews and obtain the general conclusions. Current search engines are not designed to support systematic reviews in Computer Science, Information Systems and Software Engineering (Brereton et al., 2007). Therefore, we are limited to perform source-dependent searches. Moreover, from this observation we have learned that automating tools and techniques need to be applicable for the reviews.

Another lesson learned is related to the step of performing data analysis. Most systematic reviews in Computer Science, Information Systems and Software Engineering are exploratory and based on a quantitative approach. In our study, we have calculated the frequency of complexity issues in the analysed papers. Therefore, different quantitative analysis approaches, like statistical analysis, should be used for the data analysis. However, note that a sufficiently large sample needs to be studied to apply statistical methods.

Lessons learned regarding the complexity issues and their possible solutions in FIS development are mainly presented in the form of the developed framework, which allows us to systematize found complexity issues and, consequently, their solutions. Moreover, we have determined that the found complexity issues are not new in the software systems development area. In the literature, we can find a number of general solutions for a particular issue, for example, computational complexity can be reduced through reduction and approximation techniques. Therefore, the following two strategies can be chosen to solve found complexity issues in FIS development: 1) look for existing and approved solutions in the more general field, or 2) invent and approve new solutions for FIS development.

\section{Conclusions}

The analysis of the related works in the field of FIS development shows that there is plenty of approaches to develop FIS. Their authors point out various limitations, issues, or drawbacks of developing FIS because of its complexity. All those complexity issues complicate the automatic FIS development and need to be solved. In the literature, they are not investigated sufficiently in a systematic and comprehensive way. Consequently, there is a need to determine what complexity issues exist in FIS development and which solutions are applied to solve them.

To answer the defined research questions, we have applied SLR and SMS with a keyword map review. SLR allows us to perform an in-depth analysis. SMS with a keyword map is employed to visualize and better understand each concept's real meaning in FIS development, systematize existing solutions of identified complexity issues, and develop the framework of complexity issues and their possible solutions in FIS development. 
The obtained analysis results of RQ1 show four main complexity issues: 1) computational complexity, 2) complexity of fuzzy rules, 3) complexity of MF development, and 4) data complexity. The occurrence of those complexity issues in the analysed papers are the following: the complexity of fuzzy rules have the highest occurrence, the complexity of MF development - high occurrence, computational complexity - moderate occurrence, and data complexity - low occurrence. The obtained analysis results of RQ2 show that the same solutions, like genetic algorithms, neural networks, approximation and optimization techniques, etc., are used to solve different complexity issues.

Based on the found answers to the defined research questions, we have proposed a framework of complexity issues and their possible solutions in FIS development. It allows a better understanding of FIS development complexity issues and their possible solutions for the developers. It encourages further research directions on a more effective and efficient FIS development by researchers.

In future research, we are going to do the following:

1) extend our review to several search sources;

2) extend our research to examine the synergy effect in FIS;

3) apply other techniques, like clustering analysis, etc., for co-relationship analysis of complexity issues and their solutions; and

4) analyse FIS development and deep learning possibilities.

\section{References}

Aghaeipoor, F., Eftekhari, M. (2019). EEFR-R: extracting effective fuzzy rules for regression problems, through the cooperation of association rule mining concepts and evolutionary algorithms. Soft Computing, 23(22), 11737-11757.

Aghaeipoor, F., Javidi, M. (2019). MOKBL+MOMs: an interpretable multi-objective evolutionary fuzzy system for learning high-dimensional regression data. Information Sciences, 496, 1-24.

Akbarzadeh-T, M.R., Kumbla, K., Tunstel, E., Jamshidi, M. (2000). Soft computing for autonomous robotic systems. Computers \& Electrical Engineering, 26(1), 5-32.

Alaei, H., Salahshoor, K., Alaei, H. (2013). A new integrated on-line fuzzy clustering and segmentation methodology with adaptive PCA approach for process monitoring and fault detection and diagnosis. Soft Computing, 17(3), 345-362.

Alcalá, R., Alcalá-Fdez, J., Herrera, F. (2007). A proposal for the genetic lateral tuning of linguistic fuzzy systems and its interaction with rule selection. IEEE Transactions on Fuzzy Systems, 15(4), 616-635.

Alcalá, R., Alcalá-Fdez, J., Gacto, M., Herrera, F. (2009a). Improving fuzzy logic controllers obtained by experts: a case study in HVAC systems. Applied Intelligence, 31(1), 15-30.

Alcalá, R., Ducange, P., Herrera, F., Lazzerini, B., Marcelloni, F. (2009b). A multi-objective evolutionary approach to concurrently learn rule and data bases of linguistic fuzzy-rule-based systems. IEEE Transactions on Fuzzy Systems, 17(5), 1106-1122.

Almasi, O.N., Rouhani, M. (2016). A new fuzzy membership assignment and model selection approach based on dynamic class centers for fuzzy SVM family using the firefly algorithm. Turkish Journal of Electrical Engineering \& Computer Sciences, 24, 1797-1814.

Altilio, R., Rosato, A., Panella, M. (2018). A sparse Bayesian model for random weight fuzzy neural networks. In: 2018 IEEE International Conference on Fuzzy Systems (FUZZ-IEEE), Rio de Janeiro, pp. 1-7. https://doi.org/10.1109/FUZZ-IEEE.2018.8491645.

Ang, K., Quek, C. (2005). RSPOP: rough set-based pseudo outer-product fuzzy rule identification algorithm. Neural Computation, 17(1), 205-243. 
Antonelli, M., Ducange, P., Lazzerini, B., Marcelloni, F. (2010). Exploiting a three-objective evolutionary algorithm for generating Mamdani fuzzy rule-based systems. In: International Conference on Fuzzy Systems, Barcelona, 2010, pp. 1-8. https://doi.org/10.1109/FUZZY.2010.5583965.

Antonelli, M., Ducange, P., Lazzerini, B., Marcelloni, F. (2011). Learning knowledge bases of multi-objective evolutionary fuzzy systems by simultaneously optimizing accuracy, complexity and partition integrity. Soft Computing, 15, 2335-2354.

Antonelli, M., Ducange, P., Marcelloni, F. (2013). An efficient multi-objective evolutionary fuzzy system for regression problems. International Journal of Approximate Reasoning, 54(9), 1434-1451.

Antonelli, M., Ducange, P., Marcelloni, F., Segatori, A. (2016). On the influence of feature selection in fuzzy rule-based regression model generation. Information Sciences, 329, 649-669.

Askari, S. (2017). A novel and fast MIMO fuzzy inference system based on a class of fuzzy clustering algorithms with interpretability and complexity analysis. Expert Systems with Applications, 84, 301-322.

Balazs, K., Koczy, L. (2012). Hierarchical-interpolative fuzzy system construction by genetic and bacterial memetic programming approaches. International Journal of Uncertainty, Fuzziness and Knowledge-Based Systems, 20(supp02), 105-131.

Banaeian, N., Nielsen, I.E., Mobli, H., Fahimnia, B. (2018). Green supplier selection using fuzzy group decision making methods: a case study from the agri-food industry. Computers \& Operations Research, 89, 337-347.

Barsacchi, M., Bechini, A., Ducange, P., Marcelloni, F. (2019). Optimizing partition granularity, membership function parameters, and rule bases of fuzzy classifiers for big data by a multi-objective evolutionary approach. Cognitive Computation, 11(3), 367-387.

Battram, A. (1998). Navigating Complexity: The Essential Guide to Complexity Theory in Business and Management. The Industrial Society.

Benigni, A., Ponci, F., Monti, A. (2012). Toward an uncertainty-based model level selection for the simulation of complex power systems. IEEE Systems Journal, 6(3), 564-574.

Bouchachia, A., Vanaret, C. (2014). GT2FC: an online growing interval type-2 self-learning fuzzy classifier. IEEE Transactions on Fuzzy Systems, 22(4), 999-1018.

Brereton, P., Kitchenham, B.A., Budgen, D., Turner, M., Khalil, M. (2007). Lessons from applying the systematic literature review process within the software engineering domain. Journal of Systems and Software, 80(4), 571-583.

Casillas, J., Cordón, O., del Jesus, M., Herrera, F. (2005). Genetic tuning of fuzzy rule deep structures preserving interpretability and its interaction with fuzzy rule set reduction. IEEE Transactions on Fuzzy Systems, 13(1), 13-29.

Cavalieri, S., Russo, M. (1998). Improving Hopfield neural network performance by fuzzy logic-based coefficient tuning. Neurocomputing, 18(1-3), 107-126.

Chang, X., Wang, Q., Liu, Y., Wang, Y. (2016). Sparse regularization in fuzzy $c$-means for high-dimensional data clustering. IEEE Transactions on Cybernetics, 47(9), 2616-2627.

Chan, K.Y., Aydin, M.E., Seker, H., Palade, V., Hong, T.-P. (2018). Editorial message: special issue on efficient fuzzy systems for mining large scale, imprecise, uncertain and vague data. International Journal of Fuzzy Systems, 20(4), 1203-1204.

Chao, C.T., Chen, Y.J., Teng, C.C. (1996). Simplification of fuzzy-neural systems using similarity analysis. IEEE Transactions on Systems, Man, and Cybernetics, Part B (Cybernetics), 26(2), 344-354.

Chatterjee, A., Siarry, P. (2007). A PSO-aided neuro-fuzzy classifier employing linguistic hedge concepts. Expert Systems with Applications, 33(4), 1097-1109.

Chaudhuri, A. (2014). Modified fuzzy support vector machine for credit approval classification. Ai Communications, 27(2), 189-211.

Chen, C. (2018). Eugene Garfield's scholarly impact: a scientometric review. Scientometrics, 114(2), 489-516.

Chen, C., Zhang, C. (2014). Data-intensive applications, challenges, techniques and technologies: a survey on Big Data. Information Sciences, 275, 314-347.

Chen, J., Meng, S., Zhou, W. (2019). The exploration of fuzzy linguistic research: a scientometric review based on CiteSpace. Journal of Intelligent \& Fuzzy Systems, 37(3), 3655-3669.

Chen, M. (2015). Neuro-fuzzy approach for online message scheduling. Engineering Applications of Artificial Intelligence, 38, 59-69.

Corning, P. (1998). Complexity is just a word! Technological Forecasting and Social Change, 59(2), 197-200.

Di, L., Srikanthan, T., Chandel, R., Katsunori, I. (2001). Neural-network-based self-organized fuzzy logic control for arc welding. Engineering Applications of Artificial Intelligence, 14(2), 115-124. 
Dybå, T., Dingsøyr, T. (2008). Empirical studies of agile software development: a systematic review. Information and Software Technology, 50(9-10), 833-859.

D'Urso, P. (2017). Informational paradigm, management of uncertainty and theoretical formalisms in the clustering framework: a review. Information Sciences, 400, 30-62.

Elragal, H. (2014). Mamdani and Takagi-Sugeno fuzzy classifier accuracy improvement using enhanced particle swarm optimization. Journal of Intelligent \& Fuzzy Systems, 26(5), 2445-2457.

Emami, M.R., Turksen, I.B., Goldenberg, A.A. (1998). Development of a systematic methodology of fuzzy logic modeling. IEEE Transactions on Fuzzy Systems, 6(3), 346-361.

Ephzibah, E. (2011). Time complexity analysis of genetic-fuzzy system for disease diagnosis. Advanced Computing an International Journal, 2(4), 23-31.

Eslamkhah, M., Hosseini Seno, S.A. (2019). Identifying and ranking knowledge management tools and techniques affecting organizational information security improvement. Knowledge Management Research \& Practice, 17(3), 276-305.

Fan, X., Li, C., Wang, Y. (2019). Strict intuitionistic fuzzy entropy and application in network vulnerability evaluation. Soft Computing, 23(18), 8741-8752.

Farag, W., Quintana, V., Germano Lambert-Torres, G. (1998). A genetic-based neuro-fuzzy approach for modeling and control of dynamical systems. IEEE Transactions on neural Networks, 9(5), 756-767.

Feng, H., Chen, C., Ye, F. (2006). Adaptive hyper-fuzzy partition particle swarm optimization clustering algorithm. Cybernetics and Systems, 37(5), 463-479.

Feng, S., Chen, C.P., Zhang, C.Y. (2019). A fuzzy deep model based on fuzzy restricted boltzmann machines for high-dimensional data classification. IEEE Transactions on Fuzzy Systems, 28(7), 1344-1355.

Ferdaus, M.M., Pratama, M., Anavatti, S.G., Garratt, M.A. (2019). PALM: an incremental construction of hyperplanes for data stream regression. IEEE Transactions on Fuzzy Systems, 27(11), 2115-2129.

Ferrera-Cedeño, E., Acosta-Mendoza, N., Gago-Alonso, A., García-Reyes, E. (2019). Detecting free standing conversational group in video using fuzzy relations. Informatica, 30(1), 21-32.

Firican, G. (2017). The 10 Vs of Big Data. UPSIDE where DATA means BUSINESS.

Fu, C., Lu, W., Pedrycz, W., Yang, J. (2019). Fuzzy granular classification based on the principle of justifiable granularity. Knowledge-Based Systems, 170, 89-101.

Gacto, M.J., Rafael Alcalá, R., Herrera, F. (2010). Integration of an index to preserve the semantic interpretability in the multiobjective evolutionary rule selection and tuning of linguistic fuzzy systems. IEEE Transactions on Fuzzy Systems, 18(3), 515-531.

Galende-Hernández, M., Sainz-Palmero, G., Fuente-Aparicio, M. (2012). Complexity reduction and interpretability improvement for fuzzy rule systems based on simple interpretability measures and indices by bi-objective evolutionary rule selection. Soft Computing, 16(3), 451-470.

GaneshKumar, P., Rani, C., Devaraj, D., Victoire, T. (2014). Hybrid ant bee algorithm for fuzzy expert system based sample classification. IEEE/ACM Transactions on Computational Biology and Bioinformatics, 11(2), 347-360.

Gegov, A., Arabikhan, F., Sanders, D. (2015). Rule base simplification in fuzzy systems by aggregation of inconsistent rules. Journal of Intelligent \& Fuzzy Systems, 28(3), 1331-1343.

Ge, X., Wang, P., Yun, Z. (2017). The rough membership functions on four types of covering-based rough sets and their applications. Information Sciences, 390, 1-14.

Golestaneh, P., Zekri, M., Sheikholeslam, F. (2018). Fuzzy wavelet extreme learning machine. Fuzzy Sets and Systems, 342, 90-108.

Gudas, S., Tekutov, J., Butleris, R., Denisovas, V. (2019). Modelling subject domain causality for learning content renewal. Informatica, 30(3), 455-480.

Guély, F., La, R., Siarry, P. (1999). Fuzzy rule base learning through simulated annealing. Fuzzy Sets and Systems, 105(3), 353-363.

Gusenbauer, M. (2019). Google Scholar to overshadow them all? Comparing the sizes of 12 academic search engines and bibliographic databases. Scientometrics, 118(1), 177-214.

Gusenbauer, G., Haddaway, N. (2020). Which academic search systems are suitable for systematic reviews or meta-analyses? Evaluating retrieval qualities of Google Scholar, PubMed and 26 other resources. Research Synthesis Methods, 11(2), 181-217.

Harandi, F., Derhami, V. (2016). A reinforcement learning algorithm for adjusting anteced-ent parameters and weights of fuzzy rules in a fuzzy classifier. Journal of Intelligent and Fuzzy Systems, 30(4), 2339-2347.

Hata, R., Islam, M.M., Murase, K. (2016). Quaternion neuro-fuzzy learning algorithm for generation of fuzzy rules. Neurocomputing, 216, 638-648. 
Hilletofth, P., Sequeira, M., Adlemo, A. (2019). Three novel fuzzy logic concepts applied to reshoring decisionmaking. Expert Systems with Applications, 126, 133-143.

Hong, T., Chen, J. (1999). Finding relevant attributes and membership functions. Fuzzy Sets and Systems, 103(3), 389-404.

Huang, A.F., Yang, S.J., Wang, M., Tsai, J.J. (2010). Improving fuzzy knowledge integration with particle swarmoptimization. Expert Systems with Applications, 37(12), 8770-8783.

Ibarra, L., Rojas, M., Ponce, P., Molina, A. (2015). Type-2 Fuzzy membership function design method through a piecewise-linear approach. Expert Systems with Applications: An International Journal, 42(21), 7530-7540.

Ishibuchi, H., Nojima, Y. (2009). Discussions on interpretability of fuzzy systems using simple examples. In: Proceedings of the Joint 2009 International Fuzzy Systems Association World Congress and 2009 European Society of Fuzzy Logic and Technology Conference, Lisbon, Portugal, July 20-24, 2009, pp. 1649-1654.

Ishibuchi, H., Yamamoto, T. (2003). Interpretability issues in fuzzy genetics-based machine learning for linguistic modelling. In: Lawry, J., Shanahan, J., Ralescu, L.A. (Eds.), Modelling with Words. Lecture Notes in Computer Science, Vol. 2873. Springer, Berlin, Heidelberg, pp. 209-228.

Ishibuchi, H., Nakashima, Y., Yusuke Nojima, Y. (2011). Performance evaluation of evolutionary multiobjective optimization algorithms for multiobjective fuzzy genetics-based machine learning. Soft Computing, 15(12), 2415-2434.

Ivarsson, M., Gorschek, T. (2011). A method for evaluating rigor and industrial relevance of technology evaluations. Empirical Software Engineering, 16(3), 365-395.

Jalali, S., Wohlin, C. (2012). Systematic literature studies: database searches vs. backward snowballing. In: Proceedings of the 2012 ACM-IEEE International Symposium on Empirical Software Engineering and Measurement, pp. 29-38.

Jee, T.L., Tay, K.M., Ng, C.K. (2013). A new fuzzy criterion-referenced assessment with a fuzzy rule selection technique and a monotonicity-preserving similarity reasoning scheme. Journal of Intelligent \& Fuzzy Systems, 24(2), 261-279.

Jin, X., Wah, B.W., Cheng, X., Wang, Y. (2015). Significance and challenges of big data research. Big Data Research, 2(2), 59-64.

Juang, C.F., Juang, K.J. (2012). Reduced interval type-2 neural fuzzy system using weighted bound-set boundary operation for computation speedup and chip implementation. IEEE Transactions on Fuzzy Systems, 21(3), 477-491.

Kahraman, C., Öztayşi, B., Çevik Onar, S. (2016). A comprehensive literature review of 50 years of fuzzy set theory. International Journal of Computational Intelligence Systems, 9(sup1), 3-24.

Karnik, N., Mendel, J. (2001). Operations on type-2 fuzzy sets. Fuzzy Sets and Systems, 122(2), 327-348.

Kaynak, O., Jezernik, K., Szeghegyi, A. (2002). Complexity reduction of rule based models: a survey. In: 2002 IEEE World Congress on Computational Intelligence. 2002 IEEE International Conference on Fuzzy Systems. FUZZ-IEEE'O2. Proceedings (Cat. No. 02CH37291), Vol. 2, Honolulu, HI, USA, pp. 1216-1221. https://doi.org/10.1109/FUZZ.2002.1006677.

Khalil, G.M., Gotway Crawford, C.A. (2015). A bibliometric analysis of US-based research on the behavioral risk factor surveillance system. American Journal of Preventive Medicine, 48(1), 50-57.

Kim, M.S., Kim, C.H., Lee, J.J. (2005). Evolving structure and parameters of fuzzy models with interpretable membership functions. Journal of Intelligent \& Fuzzy Systems, 16(2), 95-105.

Kim, M.S., Kim, C.H., Lee, J.J. (2006a). Evolving compact and interpretable Takagi-Sugeno fuzzy models with a new encoding scheme. IEEE Transactions on Systems, Man, and Cybernetics, Part B (Cybernetics), 36(5), 1006-1023.

Kim, M.W., Khil, A., Ryu, J.W. (2006b). Efficient fuzzy rules for classification. In: 2006 International Workshop on Integrating AI and Data Mining, Hobart, Tas., 2006, pp. 50-57. https://doi.org/10.1109/AIDM.2006.5.

Kitchenham, B. (2004). Procedures for performing systematic reviews. Joint Technical Report TR/SE-0401, pp. 1-26.

Kitchenham, B., Charters, S. (2007). Guidelines for Performing Systematic Literature Reviews in Software Engineering. Technical Report.

Kitchenham, B., Pearl Brereton, O., Budgen, D., Turner, M., Bailey, J., Linkman, S. (2009). Systematic literature reviews in software engineering - a systematic literature review. Information and Software Technology, 51(1), $7-15$.

Kitchenham, B., Budgen, D., Brereton, O. (2011). Using mapping studies as the basis for further research-a participant-observer case study. Information and Software Technology, 53(6), 638-651. 
Kóczy, L., Hirota, K. (1993). Approximate reasoning by linear rule interpolation and general approximation. International Journal of Approximate Reasoning, 9(3), 197-225.

Koczy, L.T., Hirota, K. (1997). Size reduction by interpolation in fuzzy rule bases. IEEE Transactions on Systems, Man, and Cybernetics, Part B (Cybernetics), 27(1), 14-25.

Kondratenko, Y.P., Klymenko, L.P., Zu'bi E. Y. M, A. (2013). Structural optimization of fuzzy systems' rules base and aggregation models. Kybernetes, 42(5), 831-843.

Lee, C.-H., Pan, H.-Y. (2009). Performance enhancement for neural fuzzy systems using asymmetric membership functions. Fuzzy Sets and Systems, 160(7), 949-971.

Lee, C.H., Li, C.T., Chang, F.Y. (2011). A species-based improved electromagnetism-like mechanism algorithm for TSK-type interval-valued neural fuzzy system optimization. Fuzzy Sets and Systems, 171(1), $22-43$.

Lee, C., Wang, M., Lan, S. (2014). Adaptive personalized diet linguistic recommendation mechanism based on type-2 fuzzy sets and genetic fuzzy markup language. IEEE Transactions on Fuzzy Systems, 23(5), 1777-1802.

Lee, R. (2019). Chaotic interval type-2 fuzzy neuro-oscillatory network (CIT2-FNON) for Worldwide 129 financial products prediction. International Journal of Fuzzy Systems, 21(7), 2223-2244.

Leng, G., Zeng, X., Keane, J. (2009). A hybrid learning algorithm with a similarity-based pruning strategy for self-adaptive neuro-fuzzy systems. Applied Soft Computing, 9(4), 1354-1366.

Lin, C.T., Pal, N.R., Wu, S.-L., Liu, Y.-T., Lin, Y.-Y. (2015). An interval type-2 neural fuzzy system for online system identification and feature elimination. IEEE Transactions on Neural Networks and Learning Systems, 26(7), 1442-1455.

Linnenluecke, M., Marrone, M., Singh, A. (2019). Conducting systematic literature reviews and bibliometric analyses. Australian Journal of Management, 45(2), 175-194.

Liu, H., You, J., Li, Z., Tian, G. (2017). Fuzzy Petri nets for knowledge representation and reasoning: a literature review. Engineering Applications of Artificial Intelligence, 60, 45-56.

Li, X., Shen, G.Q., Wu, P., Wang, X. (2017). Mapping the knowledge domains of Building Information Modeling (BIM): a bibliometric approach. Automation in Construction, 84, 195-206.

Mallett, R., Hagen-Zanker, J., Slater, R., Duvendack, M. (2012). The benefits and challenges of using systematic reviews in international development research. Journal of Development Effectiveness, 4(3), 445-455.

Mamdani, E. (1974). Application of fuzzy algorithms for control of simple dynamic plant. Proceedings of the Institution of Electrical Engineers, 121(12), 1585-1588.

Marimuthu, P., Perumal, V., Vijayakumar, V. (2016). OAFPM: optimized ANFIS using frequent pattern mining for activity recognition. The Journal of Supercomputing, 75(8), 1-20.

Martín-Martín, A., Orduna-Malea, E., Thelwall, M., López-Cózar, E. (2018). Google Scholar, Web of Science, and Scopus: a systematic comparison of citations in 252 subject categories. Journal of Informetrics, 12(4), 1160-1177.

Marz, N., Warren, J. (2015). Big Data: Principles and Best Practices of Scalable Real-Time Data Systems. 1st ed. pp. 308.

Matarazzo, B., Munda, G. (2001). New approaches for the comparison of LR fuzzy numbers: a theoretical and operational analysis. Fuzzy Sets and Systems, 118(3), 407-418.

Melin, P., Ontiveros-Robles, E., Gonzalez, C.I., Castro, J.R., Castillo, O. (2019). An approach for parameterized shadowed type-2 fuzzy membership functions applied in control applications. Soft Computing, 23(11), 3887-3901.

Mesiarová-Zemánková, A., Ahmad, K. (2012). Differences between t-norms in fuzzy control. International Journal of Intelligent Systems, 27(7), 662-679.

Miliauskaite, J., Kalibatiene, D. (2020a). Complexity issues in data-driven fuzzy inference systems: systematic literature review. In: Proceedings in the International Baltic Conference on Databases and Information Systems, pp. 190-204.

Miliauskaite, J., Kalibatienè, D. (2020b). On general framework of type-1 membership function construction: case study in QoS planning. International Journal of Fuzzy Systems, 22(2), 504-521.

Mirko, S., Stjepanović, A., Stjepanović, Đ. (2019). ANFIS model for the prediction of generated electricity of photovoltaic modules. Decision Making: Applications in Management and Engineering, 2(1), 35-48.

Nguyen, N.N., Zhou, W.J., Chai Quek, C. (2015). GSETSK: a generic self-evolving TSK fuzzy neural network with a novel Hebbian-based rule reduction approach. Applied Soft Computing, 35, 29-42.

Odell, J. (2002). Agents and complex systems. Journal of Object Technology, 1(2), 35-45.

Olufunke, O.O., Ayo, C., Abraham, A., Uwadia, C. (2013). A fuzzy-mining approach for solving rule based expert system unwieldiness in medical domain. Neural Network World, 23(5), 435-450. 
Pal, T., Pal, N.R., Pal, M. (2003). Learning fuzzy rules for controllers with genetic algorithms. International Journal of Intelligent Systems, 18(5), 569-592.

Petersen, K., Vakkalanka, S., Kuzniarz, L. (2015). Guidelines for conducting systematic mapping studies in software engineering: an update. Information and Software Technology, 64, 1-18.

Pratama, M., Joo Er, M., Li, X., Oentaryo, R.J., Lughofer, E., Arifin, I. (2013). Data driven modeling based on dynamic parsimonious fuzzy neural network. Neurocomputing, 110, 18-28.

Rajab, S., Sharma, V. (2018). A review on the applications of neuro-fuzzy systems in business. Artificial Intelligence Review, 49(4), 481-510.

Rajeswari, A., Deisy, C. (2019). Fuzzy logic based associative classifier for slow learners prediction. Journal of Intelligent and Fuzzy Systems, 36(3), 2691-2704.

Ramaki, A., Rasoolzadegan, A., Bafghi, A. (2018). A systematic mapping study on intrusion alert analysis in intrusion detection systems. ACM Computing Surveys, 51(3), 1-41.

Ramathilaga, S., Jiunn-Yin Leu, J., Huang, K., Huang, Y. (2014). Two novel fuzzy clustering methods for solving data clustering problems. Journal of Intelligent and Fuzzy Systems, 26(2), 705-719.

Ravi, C., Khare, N. (2018). BGFS: design and development of brain genetic fuzzy system for data classification. Journal of Intelligent and Fuzzy Systems, 27(2), 231-247.

Renhou, L., Yi, Z. (1996). Fuzzy logic controller based on genetic algorithms. Fuzzy Sets and Systems, 83(1), $1-10$.

Rey, M., Galende, M., Fuente, M.J., Sainz-Palmero, G. (2012). Checking orthogonal transformations and genetic algorithms for selection of fuzzy rules based on interpretability-accuracy concepts. International Journal of Uncertainty, Fuzziness and Knowledge-Based Systems, 20(supp02), 159-186.

Rojas, I., Pomares, H., Ortega, J., Prieto, A. (2000). Self-organized fuzzy system generation from training examples. IEEE Transactions on Fuzzy Systems, 8(1), 23-36.

Ruiz-Garcia, G., Hagras, H., Pomares, H., Rojas, I. (2019). Towards a fuzzy logic system based on general forms of interval type-2 fuzzy sets. IEEE Transactions on Fuzzy Systems, 27(12), 2381-2395.

Sanchez-Roger, M., Oliver-Alfonso, M.D., Sanchís-Pedregosa, C. (2017). Fuzzy logic and its uses in finance: a systematic review exploring its potential to deal with banking crises. Mathematics, 7(11), 1091.

Sanz, J., Fernández, A., Bustince, H., Herrera, F. (2011). A genetic tuning to improve the performance of fuzzy rule-based classification systems with interval-valued fuzzy sets: degree of ignorance and lateral position. International Journal of Approximate Reasoning, 52(6), 751-766.

Sanz, J., Bustince, H., Fernández, A., Herrera, F. (2012). IIVFDT: Ignorance functions based interval-valued fuzzy decision tree with genetic tuning. International Journal of Uncertainty, Fuzziness and KnowledgeBased Systems, 20(supp02), 1-30.

Shahidah, N., Azni, A.H., Alwi, N., Seman, K., Bakar, N.H. (2017). Systematic literature review: correlated fuzzy logic rules for node behavior detection in wireless sensor network. Journal of Telecommunication, Electronic and Computer Engineering (JTEC), 9(3-3), 109-115.

Shahparast, H., Mansoori, E. (2019). Developing an online general type-2 fuzzy classifier using evolving type-1 rules. International Journal of Approximate Reasoning, 113, 336-353.

Shakeel, Y., Krüger, J., Lausberger, C., von Nostitz-Wallwitz, I. (2018). Literature analysis-threats and experiences. In: Proceedings of the IEEE/ACM 13th International Workshop on Software Engineering for Science (SE4Science), pp. 20-27.

Soua, B., Borgi, A., Tagina, M. (2013). An ensemble method for fuzzy rule-based classification systems. Knowledge and Information Systems, 36(2), 385-410.

Stavrakoudis, D.G., Galidaki, G., Gitas, I., Theocharis, J. (2012). Reducing the complexity of genetic fuzzy classifiers in highly-dimensional classification problems. International Journal of Computational Intelligence Systems, 5(2), 254-275.

Takagi, T., Sugeno, M. (1985). Fuzzy identification of systems and its application to modeling and control. IEEE Transactions on Systems, Man, and Cybernetics, 1(SMC-15), 116-132.

Thirunarayan, K., Sheth, A.P. (2013). Semantics-empowered approaches to big data processing for physicalcyber-social applications. Semantics for Big Data AAAI Technical Report FS-13-04, In AAAI Fall Symposium Series, (2013, November), pp. 1-8.

Tikk, D., Baranyi, P. (2000). Comprehensive analysis of a new fuzzy rule interpolation method. IEEE Transactions on Fuzzy Systems, 8(3), 281-296.

van Eck, N., Waltman, L., Noyons, E., Buter, R. (2010). Automatic term identification for bibliometric mapping. Scientometrics, 82(3), 581-596. 
Vilutiene, T., Kalibatiene, D., Reza Hosseini, M., Pellicer, E., Zavadskas, E.K. (2019). Building Information Modeling (BIM) for structural engineering: a bibliometric analysis of the literature. Advances in Civil Engineering, 2019, 5290690. https://doi.org/10.1155/2019/5290690.

Waldrop, M.M. (1993). Complexity: The Emerging Science at the Edge of Order and Chaos.

Waltman, L., Van Eck, N.J. (2013). A smart local moving algorithm for large-scale modularity-based community detection. The European Physical Journal B, 86(11), 471.

Wang, H., Kwong, S., Jin, Y., Wei, W., Man, K.F. (2005). Multi-objective hierarchical genetic algorithm for interpretable fuzzy rule-based knowledge extraction. Fuzzy sets and systems, 149(1), 149-186.

Wang, H., Xu, Z., Pedrycz, W. (2017). An overview on the roles of fuzzy set techniques in big data processing: trends, challenges and opportunities. Knowledge-Based Systems, 118, 15-30.

Wu, X., Zhu, X. (2008). Mining with noise knowledge: error-aware data mining. IEEE Transactions on Systems, Man, and Cybernetics-Part A: Systems and Humans, 38(4), 917-932.

Yen, J., Wang, L. (1999). Simplifying fuzzy rule-based models using orthogonal transformation methods. IEEE Transactions on Systems, Man, and Cybernetics, Part B (Cybernetics), 29(1), 13-24.

Zhao, W., Zhang, J., Li, K. (2015). An efficient LS-SVM-based method for fuzzy system construction. IEEE Transactions on Fuzzy Systems, 23(3), 627-643.

Zhu, X., Pedrycz, W., Li, Z. (2017). Granular representation of data: a design of families of $\epsilon$-information granules. IEEE Transactions on Fuzzy Systems, 26(4), 2107-2119.

D. Kalibatienè is a full-time professor at the Department of Information Systems in Vilnius Gediminas Technical University (VilniusTECH). She received her doctoral degree in technological sciences, informatics engineering (PhD) in 2009 (the topic of doctoral dissertation is Ontology-Based Development of Domain Rules), and received the assoc. prof. degree in 2013. She is a co-author of more than 40 research papers and one book (Advanced Databases) in Computer Sciences. Her research interests include business rules and ontology-based information system development and conceptual modelling; knowledge-based multi-criteria dynamic business process modelling and simulation; multi-criteria decision-making method application in different fields; fuzzy theory application in quality planning and prediction.

She delivered lectures at Palermo University (Sicily, Italy), University of La Laguna (Tenerife), and University of Rousse (Bulgaria). She is supervising three doctoral students. She participated in the High Technology Development Program Project "Business Rules Solutions for Information Systems Development" (VeTIS). She is a member of the European Committee and Lithuanian Government supported SOCRATES/ERASMUS Thematic Network project "Doctoral Education in Computing" (ETN DEC), "Teaching, Research, Innovation in Computing Education" (ETN TRICE) and "Future Education and Training in Computing: How to Support Learning at Anytime Anywhere" (ETN FETCH). She participated in preparing the bachelor study programme "Business Information Systems" (now "Information Systems") in 2010; and the bachelor study programme "Software Engineering" in 2012. Since 2019, she is a chair of the Study Program Committee of the "Information Systems Software Engineering" master study programme. Also, she is a member of the Study Program Committee of the bachelor study programmes "Information Systems" and "Software Engineering". 
J. Miliauskaitė is a junior researcher at Vilnius University (Lithuania) Institute of Data Science and Digital Technologies Department of Cyber-Social Systems Engineering Group. She defended her a $\mathrm{PhD}$ in technological sciences, informatics engineering at Vilnius University (2015) on the topic A Fuzzy Inference-Based Approach to Planning Quality of Enterprise Business Services. Her research interests include enterprise business services, service-oriented enterprise systems, web service composition, quality of service modelling and evaluation in service-oriented enterprise systems. She is a co-author of research papers in the field of computer sciences. She is a lecturer at Vilnius University Faculty of Mathematics and Informatics and Vilnius Gediminas Technical University (Lithuania) Faculty of Fundamental Sciences Department of Information Systems. She participated in the project of EU Structural Funds "Theoretical and Engineering Aspects of E-Service Technology Development and Application in High-Performance Computing Platforms". She is a member of organising committee of the International Baltic Conference on Databases and Information Systems (Baltic DB\&IS 2012, Baltic DB\&IS 2018). She is a member of Lithuanian Computer Society (LIKS). 